\title{
Recent Development and Perspectives of Flexible Zinc-Air Batteries
}

\author{
Hao-Tian Teng ${ }^{1,2}$, Wen-Tao Wang ${ }^{1,2}$, Xiao-Feng Han ${ }^{1,2}$, Xiang Hao ${ }^{3}$, Ruizhi Yang ${ }^{1,2,{ }^{*},}$ \\ Jing-Hua Tian $1,2,4,{ }^{*}$ \\ ${ }^{1}$ College of Energy, Soochow Institute for Energy and Materials InnovationS, Collaborative Innovation Center of Suzhou Nano \\ Science and Technology, Soochow University, Suzhou 215006, Jiangsu Province, China. \\ ${ }^{2}$ Key Laboratory of Advanced Carbon Materials and Wearable Energy Technologies of Jiangsu Province, Soochow University, \\ Suzhou 215006, Jiangsu Province, China. \\ ${ }^{3}$ School of Physical Science and Technology, Suzhou University of Science and Technology, Suzhou, 215009, \\ Jiangsu Province, China. \\ ${ }^{4}$ Fujian Science \& Technology Innovation Laboratory for Energy Materials of China (Tan Kah Kee Innovation Laboratory), Xiamen, \\ 361104, Fujian Province, China.
}

Abstract: In recent years, flexible and wearable electronic devices have attracted increasing research, industrial, and consumer attention. In particular, flexible zinc-air batteries (ZABs) are expected to become a promising power supply source for next-generation electronic products, especially the flexible and wearable ones, because of their high theoretical energy

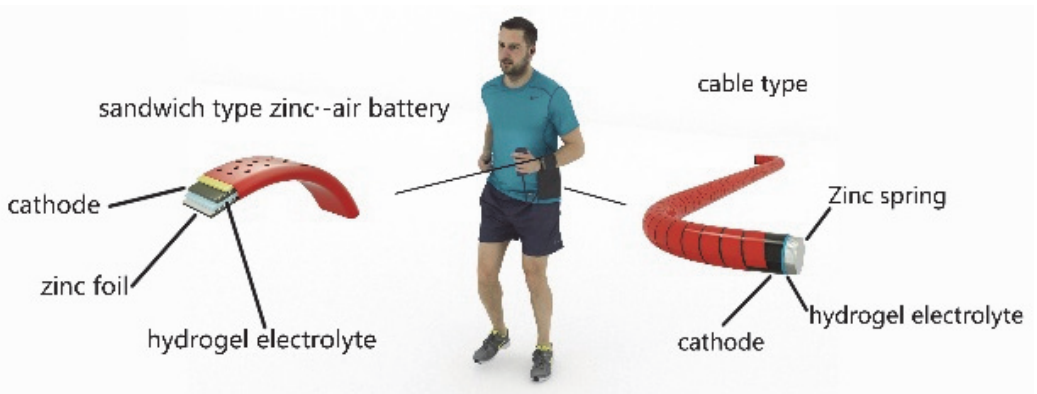
density, high specific capacity, high safety, and adaptability to uneven surfaces like human body. In the research field of flexible ZABs, a steady progress has been observed, and various ZAB preparation methods have been recently proposed. In this review, the main achievements and limitations of the recent research related to flexible ZABs are described. Firstly, the importance and applications of ZABs are discussed, followed by the working principle and configuration of typical ZABs. In the main text, the recent development of gel electrolytes, anodes, and cathodes is reviewed in detail. Currently, one of the most important limitations in the preparation of high-performance ZABs is the selection or preparation of a suitable gel electrolyte. A good gel electrolyte should have the ability of high-water holding capacity, high and low temperature resistance, high $\mathrm{CO}_{2}$-tolerance, excellent ionic conductivity, and good mechanical ductility. Several gel electrolytes with various functions have been developed. However, novel gel electrolytes with multifunctional properties have not been developed. In addition, interfaces between the gel electrolyte and air cathode and those between the gel electrolyte and metal anode must be investigated in detail for ZAB performance improvement. Till now, only the effects of physical compression on the electrolyte-air cathode and electrolyte-metal anode interfaces have been adopted and investigated. Moreover, the air cathode and metal anode must exhibit high flexibility to expand the application scope of ZABs as flexible power supplies. Carbon cloth has been typically used as the substrate of the air cathode; however, carbon corrosion occurs under high potential, which needs to be overcome. Meanwhile, the use of nickel mesh or copper foam as the substrate for the cathode will make the flexible ZABs too rigid and not bendable. For the metal anode, mostly zinc sheet or zinc spring have been used to meet the demand of flexibility. However, if novel strategies for the development of doped zinc anodes are investigated, such as those based on the utilization of zinc powder-metal combination, ZAB performance will be significantly improved. If the above-mentioned limitations are overcome, flexible ZABs will not be limited to laboratory use, and can be widely applied in commercial

Received: July 6, 2021; Revised: August 7, 2021; Accepted: August 19, 2021; Published online: August 26, 2021.

*Corresponding authors. Emails: yangrz@suda.edu.cn (R.Y.); jhtian@suda.edu.cn (J.T.).

The project was supported by the National Key R\&D Program of China (2020YFB1505703) and the National Natural Science Foundation of China (21673153, $51972220,51572181)$. 
wearable electronic products. Furthermore, the challenges and future perspectives of ZABs are discussed in this review.

Key Words: Gel electrolyte; Metal anode; Air cathode; Battery configuration; Flexible zinc-air battery

\section{柔性锌-空气电池进展与展望}

滕浩天 ${ }^{1,2}$, 王文涛 1,2 , 韩晓峰 1,2 , 郝翔 3 ，杨瑞枝 $1,2,{ }^{*}$ ，田景华 1,2,4,

1 苏州大学能源学院, 能源与材料创新研究院, 苏州纳米科技协同创新中心, 江苏苏州 215006

2 苏州大学江苏省先进碳材料与可穿戴技术重点实验室, 江苏苏州 215006

3 苏州科技大学物理科学与技术学院, 江苏苏州 215009

4 中国福建能源材料科学与技术创新实验室(嘉庚创新实验室), 福建 厦门 361104

摘要: 近年来, 人们越来越关注柔性可穿戴电子设备。柔性锌-空气电池由于有较高的理论能量密度以及对像人体一样不 均匀表面的适应能力, 有望成为下一代电子产品的电源。在柔性锌-空气电池研究领域, 人们已经取得了较好的研究进 展, 各种柔性锌-空气电池的制备方法已被报道。本文阐述了近年来柔性锌-空气电池的主要成就以及面临的困难, 特别 是关注凝胶电解质、金属阳极以及柔性空气阴极对柔性锌-空气电池电化学性能的影响, 最后讨论了柔性锌-空气电池面 临的主要挑战与发展前景。

关键词: 凝胶电解质; 金属阳极; 空气阴极; 电池构型; 柔性锌-空气电池 中图分类号: 0646

\section{1 引言}

随着科技的高速发展, 人们对于柔性可穿戴 电子产品及柔性电源的需求也越来越高。作为柔 性电源, 锂离子电池是一个不错的选择, 它有着较 高的理论能量密度 $\left(387 \mathrm{Wh} \cdot \mathrm{kg}^{-1}\right.$, 石墨/钴酸锂体 系) 1 。但由于其使用了可燃性的有机电解质, 具有 一定的安全隐患, 很难在柔性电子器件, 特别是与 人体接触相关的柔性电子器件上大规模应用。此 外, 由于锂离子电池制造要求严格, 以及锂资源有 限等问题, 人们开始为柔性电子器件的电源寻找 替代产品。锌-空气电池由于其自身更高的理论能 量密度 $\left(1086 \mathrm{Wh} \cdot \mathrm{kg}^{-1}\right)$ 、安全性高 ${ }^{2}$, 因而展现出替 代锂离子电池作为柔性电源的巨大潜力。它可以 作为可充电织物, 为穿戴设备如电子手表、手机等 电子产品供电(图1) ${ }^{3}$ 。

柔性锌-空气电池由凝胶电解质、锌阳极以及 空气阴极组成。电解质自身柔韧性以及与空气阴 极和锌阳极的贴合性、锌阳极和空气阴极自身的 㓞性等对能否实现柔性的锌-空气电池起着决定 性作用。与此同时, 还需要考虑电解质的离子电导 率、保水性, 阴极催化剂的电催化性能以及锌阳极 的枝晶生长等因素对锌-空气电池性能的影响。

本篇文章首先介绍了传统锌-空气电池的基 本工作原理、柔性锌-空气电池常见构型, 重点关 注了凝胶电解质种类、柔性锌阳极以及空气阴极
的改善对柔性锌-空气电池性能的影响, 最后对该 领域的未来发展方向进行了展望。

\section{2 锌-空气电池的工作原理}

传统的锌-空气电池结构如图 2 所示 ${ }^{4}$, 由空气 阴极、电解液以及锌阳极组成。它的放电反应过程 如下:

$$
\begin{aligned}
& \text { 锌阳极: } \mathrm{Zn}+4 \mathrm{OH}^{-} \leftrightarrow \mathrm{Zn}(\mathrm{OH})_{4}^{2-}+2 \mathrm{e}^{-} \\
& \mathrm{Zn}(\mathrm{OH})_{4}^{2-} \leftrightarrow \mathrm{ZnO}+\mathrm{H}_{2} \mathrm{O}+2 \mathrm{OH}^{-} \\
& \text {空气阴极: } \mathrm{O}_{2}+2 \mathrm{H}_{2} \mathrm{O}+4 \mathrm{e}^{-} \leftrightarrow 4 \mathrm{OH}^{-}
\end{aligned}
$$

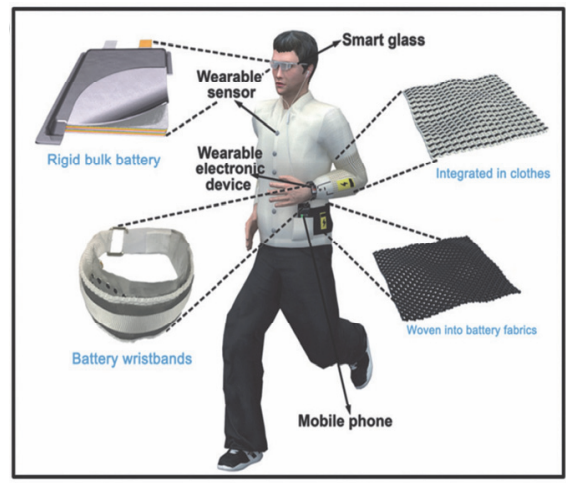

图 1 柔性可穿戴电池应用示意图 ${ }^{3}$

Fig. 1 Schematic illustration for the applications of flexible metal-air batteries ${ }^{3}$.

Adapted with permission from Ref. 3. Copyright 2020, Elsevier B.V. 


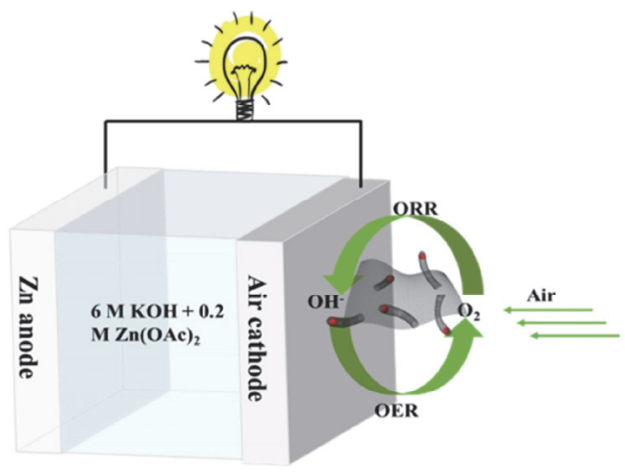

图 2 传统的锌-空气电池结构 4

Fig. 2 Schematic configuration of typical ZAB ${ }^{4}$.

Adapted with the permission from Ref. 4. Copyright 2020 American

Chemical Society.

锌离子从金属阳极上溶解析出, 与电解液中 的 $\mathrm{OH}^{-}$离子发生反应, 形成 $\mathrm{Zn}(\mathrm{OH})_{4}^{2-}$, 进一步转变 成 $\mathrm{ZnO}$ 。同时环境中的氧气在空气电极阴极催化层 中被还原成 $\mathrm{OH}^{-}$。放电过程与充电过程取决于锌阳 极上锌的剥离与沉积, 而在空气阴极上发生氧还 原反应(Oxygen Reduction Reaction, ORR)与氧析 出反应(Oxygen Evolution Reaction, OER) ${ }^{5}$ 。

对柔性锌-空气电池机械性能以及离子导电 性能起决定性作用的是凝胶电解质。柔性锌-空气 电池常用的凝胶电解质主要分为聚合物类电解 质、氧化石墨烯类电解质以及纤维素类电解质, 下
面将会对柔性锌-空气电池中常用的凝胶电解质 逐一进行介绍。

\section{3 凝胶电解质}

与传统的锌-空气电池相比, 柔性锌-空气电池 通常应用于可穿戴产品中。由于电解液容易从封 装材料中泄漏, 所以电解质需要良好的保水能力 以及拉伸能力, 因此凝胶电解质成为柔性锌-空气 电池的重要研究方向 ${ }^{6}$ 。

\section{1 聚合物类电解质}

聚合物电解质例如聚乙烯醇 (Polyvinyl alcohol (PVA), 分子式 $\left[\mathrm{C}_{2} \mathrm{H}_{4} \mathrm{O}\right]_{n}$ ) 具有良好的亲水能 力, 同时也有很好的成膜能力, 一般被人们选择为 聚合物主体。氢氧化钾 $(\mathrm{KOH})$ 有着较高的导电性, 一般作为碱性电解质的离子导体。Xu等 7 在 2015 年 首次组装并报道了柔性锌-空气电池, 制备了高离子 导电性的水凝胶电解质。他们首先将聚环氧乙烷 (Polyethylene oxide (PEO), $-\left[-\mathrm{CH}_{2}-\mathrm{CH}_{2}-\mathrm{O}-\right]_{n}$ ) 与 $\mathrm{PVA}$ 溶解于水中, 再加入 $\mathrm{KOH}$ 以提高导电性(图 3a)。成膜后的凝胶电解质具有良好的机械性能、 弯折和拉伸能力(图3b)。它甚至可以拉伸到 $300 \%$ 的长度而不断裂(图3c)。此水凝胶电解质的电阻为 $10 \Omega$, 同时引入 $\mathrm{KOH}$ 使其离子电导率达到了 0.3 $\mathrm{S} \cdot \mathrm{cm}^{-1}$ (图3d)。但是它的储水能力在文中并未提 及, 拉伸之后性能是否改变, 以及能否恢复到原来 (a)

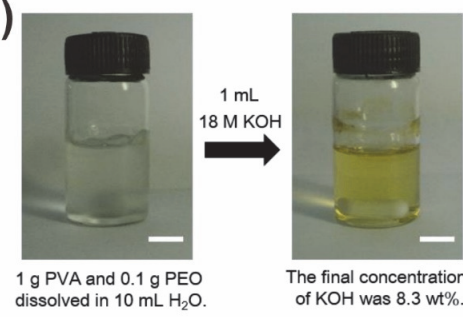

(c)

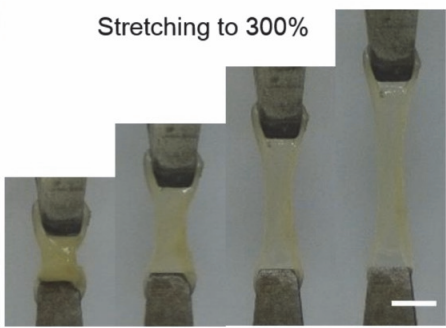

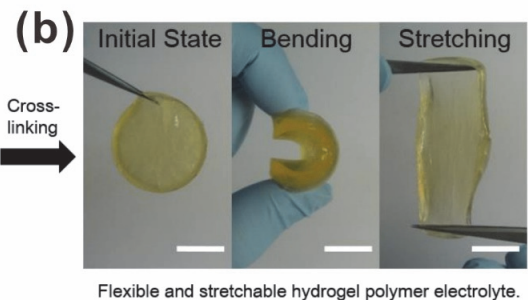

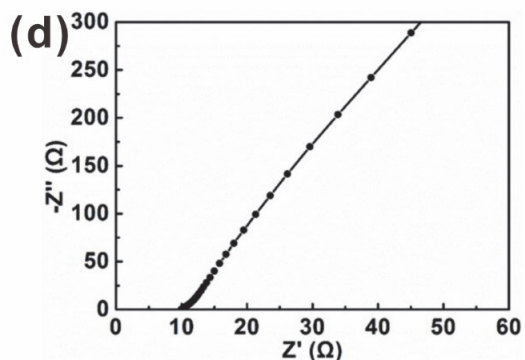

图 3 PEO-PVA 凝胶电解质的制备、机械性能及电阻测试 7

Fig. 3 Preparation and characterizations of gel polymer electrolyte ${ }^{7}$.

(a) Photographs of synthetic process of hydrogel polymer electrolyte solution, scale bar: $1 \mathrm{~cm}$; (b) photographs of free-standing hydrogel polymer electrolyte after crosslinking with initial state, bending and stretching, scale bar: $1 \mathrm{~cm}$; (c) photographs of the hydrogel polymer electrolyte in a stretching process up to $300 \%$, scale bar: $0.5 \mathrm{~cm}$; (d) alternating current $(\mathrm{AC})$ impedance spectra of the hydrogel polymer electrolyte at the frequency range from $1000 \mathrm{kHz}$ to $0.01 \mathrm{~Hz}$. 
的形状也成为一个问题。

人们也通常选择其他聚合物如聚丙烯腈 (polyacrylonitrile (PAN), $\left[\mathrm{C}_{3} \mathrm{H}_{3} \mathrm{~N}\right]_{n}$ )、聚丙烯酸 (polyacrylic acid (PAA), $\left[\mathrm{C}_{3} \mathrm{H}_{4} \mathrm{O}_{2}\right]_{n}$ ) 和聚丙烯酰胺 (polyacrylamide (PAM), $\left.\left[\mathrm{C}_{3} \mathrm{H}_{5} \mathrm{NO}\right]\right)^{4-6,8-11}$ 。但仅仅 是 PVA- $\mathrm{KOH}$ 结合或是其他聚合物与 $\mathrm{KOH}$ 结合已 经不能满足人们构建高性能柔性锌-空气电池的 需求, 例如: 高离子电导率、高保水能力、抗 $\mathrm{CO}_{2}$ 能力的需求等。通常需要引入额外的添加剂来完 善凝胶电解质的性能。如Fan等 ${ }^{12}$ 在 PVA中引入了 $\mathrm{SiO}_{2}$ 颗粒, 不仅使得凝胶电解质的离子电导率得 到强化, 还使得电解质的保水与吸水能力得到提 高。制备过程如图4a所示, 聚乙二醇(polyethylene glycol, PEG)、PVA、 $\mathrm{SiO}_{2}$ 在同一溶液中加热搅拌 后冷却, 最终形成一个固态的电解质薄膜。用质量 分数为 $5 \%$ 的 $\mathrm{SiO}_{2}$ 作为增塑剂使得PVA的无定型区 域增加, 增加了自由分子体积分数, 从而使得电解 质的吸水能力增加。同时测试表明, 引入了 $\mathrm{SiO}_{2}$ 的 电解质保水能力也得到了提高, 这是由于二氧化 硅表面的羟基有利于氢键的形成，进一步提高了 保水能力。另一方面, 由于加入 $5 \%$ 质量分数的二 氧化硅, 凝胶电解质的电导率从 $36.1 \mathrm{mS} \cdot \mathrm{cm}^{-1}$ 提高 到 $50.2 \mathrm{mS} \cdot \mathrm{cm}^{-1}$, 这是由于二氧化硅填料的加入有

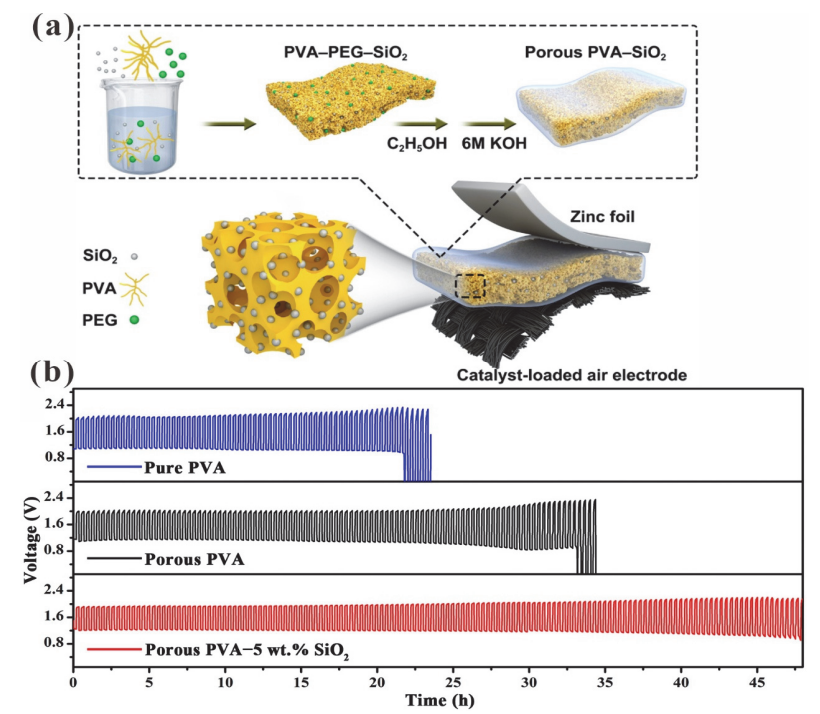

图 4 PVA-SiO 2 制备以及锌-空气电池循环能力测试 12

Fig. 4 Preparation of $\mathrm{PVA}_{-} \mathrm{SiO}_{2}$ and measurement of circulation ability of flexible zinc-air battery ${ }^{12}$.

(a) Schematic diagram of the flexible ZAB and preparation process of the porous PVA-based nanocomposite GPE along with its inner structure; (b) GCD testing with a duration of 20 min per cycle at $3 \mathrm{~mA} \cdot \mathrm{cm}^{-2}$ of the ZABs based on various PVA-based GPEs.

Adapted with permission from Ref. 12. Copyright 2020, Elsevier B.V.
助于PVA基体由晶态区域向非晶态区域的转变, 最终导致离子的迁移能力得到提高。如图 $4 \mathrm{~b}$ 所示, 由于引入了二氧化硅, 组装成的柔性锌-空气电池 循环了 144 圈 $(48 \mathrm{~h})$, 比纯PVA或多孔PVA凝胶电解 质的电池性能都要好很多。

由于锌-空气电池是个半开放体系, 空气中的 $\mathrm{CO}_{2}$ 很容易与凝胶电解质中的 $\mathrm{KOH}$ 发生反应并形 成碳酸钾, 使得凝胶电解质的离子导电能力急剧 下降。因此为了提高电解质抗 $\mathrm{CO}_{2}$ 能力, $\mathrm{Zhao}$ 等 ${ }^{13}$ 将聚环氧乙烷 $\left(\mathrm{C}_{2} \mathrm{H}_{6} \mathrm{O}_{2}\right)$ 、聚环氧丙烷 $\left(\mathrm{C}_{6} \mathrm{H}_{6} \mathrm{O}_{2}\right)$ 、聚 环氧乙烷三段共聚为聚合物电解质(记为F127)。将 采用 F 127 和 $0.5 \mathrm{~mol} \cdot \mathrm{L}^{-1} \mathrm{KOH}$ 的电解液与常用的 0.5 $\mathrm{mol} \cdot \mathrm{L}^{-1} \mathrm{KOH}$ 电解液分别组装成锌-空气电池, 并 暴露在 $\mathrm{CO}_{2}$ 氛围下进行测试。仅仅 $24 \mathrm{~h}$ 普通电池的 电解液就与二氧化碳发生剧烈反应, 而采用了 F127聚合物的电池在 $166 \mathrm{~h}$ 后其开路电压没有发生 改变, 抗 $\mathrm{CO}_{2}$ 能力得以显著提高。这是由于 $\mathrm{F} 127$ 聚 合物黏度是 $0.5 \mathrm{~mol} \cdot \mathrm{L}^{-1} \mathrm{KOH}$ 室温下的 $10^{7}$ 倍, 而黏 度越高, $\mathrm{CO}_{2}$ 的扩散系数就越低。

人们除了要提高凝胶电解质抗 $\mathrm{CO}_{2}$ 性能外, 也 要提高电解质的离子电导率。 $\mathrm{Li}$ 等 ${ }^{14}$ 就用氢氧化四 乙铵来代替传统的氢氧化钾作为离子导体, 记为 TEAOH (Tetraethylammonium hydroxide)。TEAOHPVA凝胶电解质对比常用的 KOH-PVA, 其保水能 力、离子导电率均有明显的提升。

为了扩大柔性锌-空气电池的使用范围, 凝胶 型电解质除了要面对强碱溶液外, 还要能够在高 低温的情况下保持良好的电化学性能和机械性 能。因此, Chen等 ${ }^{15}$ 将丙三醇 (Glycerol, Gly)引入 水凝胶中, 与水形成氢键的同时还能降低它的饱 和蒸汽压, 从而研发了一种工作范围从 -20 到 $70^{\circ} \mathrm{C}$ 的凝胶电解质。他们将PAM 与 PAA 和亚甲基双丙 烯酰胺结合起来, 与此同时, 也加入了 Gly与过硫 酸铵(APS), 它呈现出的结构也是一种相互交联的 网状结构(图5a)。得到的电解质具有良好的延展性 (相比之下, 普通的PAM+PAA则不显示出延展性)。 随着 Gly的加入, 机械强度先增加到最大值而后降 低。最大应力为 $145.5 \mathrm{kPa}$, 断裂张力为 $484.4 \%$, 而 加入过多的丙三醇会导致凝胶电解质力学性能下 降。无Gly的水凝胶在 $-20{ }^{\circ} \mathrm{C}$ 会变成白色的硬板状, 而加入了 Gly的水凝胶电解质则呈现出透明、柔 软、可拉伸的状态，且没有很明显的冻结现象。因 此可以得出, Gly 可以使得水凝胶在 $0{ }^{\circ} \mathrm{C}$ 以下有高 度的柔韧性与延展性。组装的柔性锌-空气电池在 不同弯折角度下充放电极化曲线并没有明显的变 化(图 $5 \mathrm{c}, \mathrm{d})$ 。进行电池的弯折与锤击测试后, 电压 
(a)

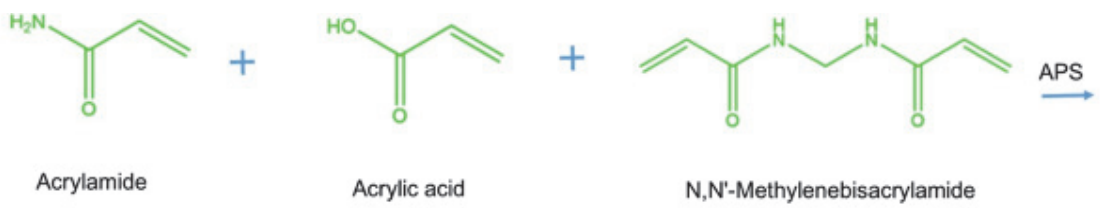

(b)

PAM/PAA Chains
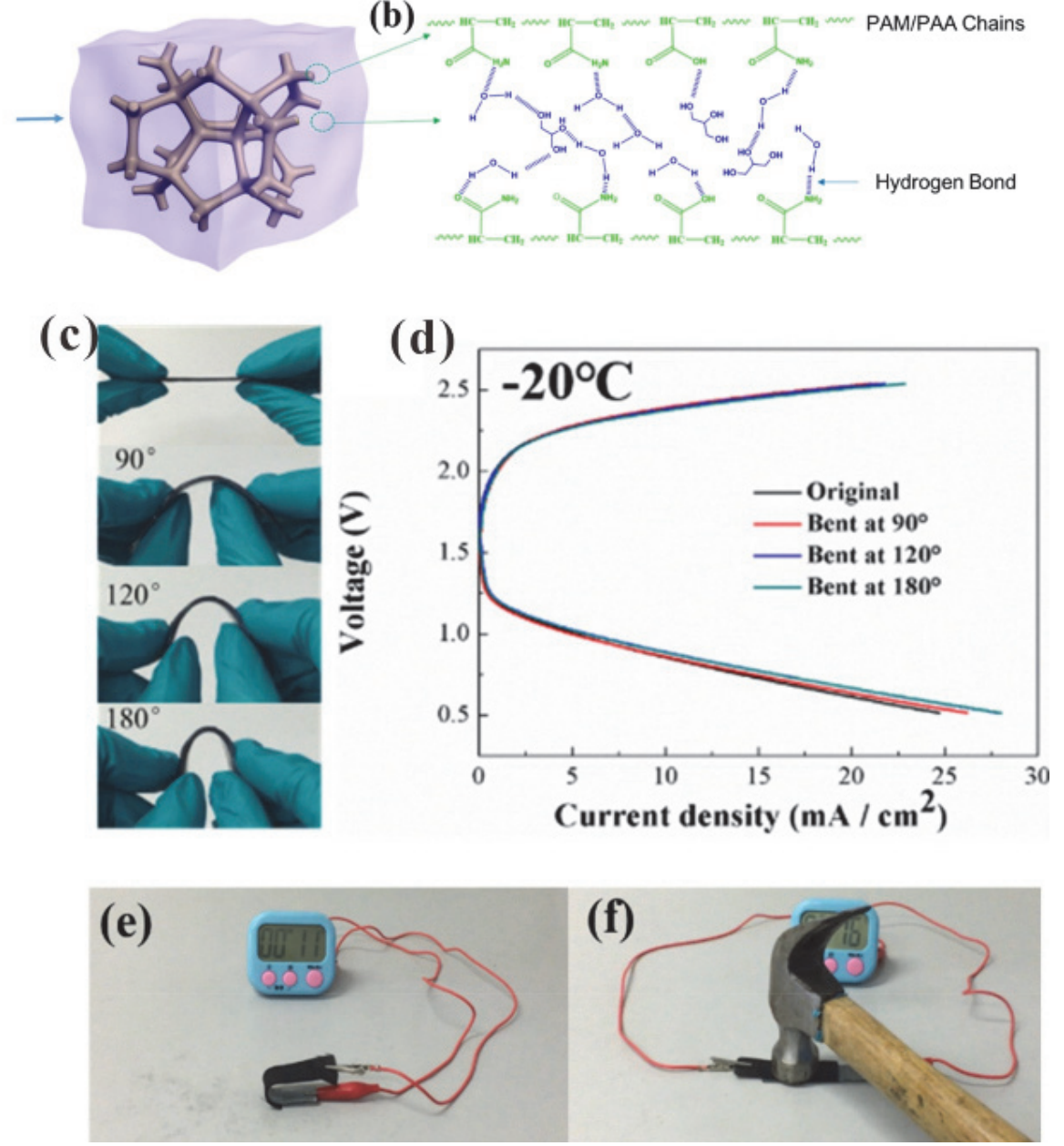

图 5 耐温水凝胶的合成及机械性能测试 15

Fig. 5 Synthesis and mechanical properties of high and low temperature resistant hydrogels ${ }^{15}$.

(a) Preparation of the temperature-tolerant hydrogel; (b) schematic illustration of the hydrogen bonding interactions taking place between Gly and the PAM/PAA polymers, and water molecules; (c) bending tests at different bending angles and (d) charge and discharge polarization curves at $-20{ }^{\circ} \mathrm{C}$;

(e, f) mechanical tests on the temperature-tolerant aqueous $\mathrm{Zn}$-air battery under various deformation conditions. Adapted with the permission from Ref. 15. Copyright 2020, American Chemical Society.

变化也较小(图5e，f)。

到目前为止，人们已经能够通过引入各种添 加剂来完善聚合物凝胶电解质的各项性能, 但是 完美的聚合物凝胶电解质还未被开发出来, 它应 该满足以下特点: 高柔韧性、高吸水能力与保水能 力、高离子导电性能、宽工作温度范围、抗 $\mathrm{CO}_{2}$ 能 力以及和电池阴阳极的良好贴合性能等。

\section{2 纤维素类凝胶电解质}

聚合物电解质由于高亲水性使得电解质的稳 定性较差, 电解质容易变形, 同时会阶段性的释放 出碱从而导致性能急剧下降。为解决上述问题, 人
们经研究发现, 纳米纤维素含有大量的羟基官能 团、柔韧性好、高的容量比以及大比表面积可以弥 补聚合物电解质的缺点。

例如, $\mathrm{Ma}$ 等 ${ }^{16}$ 研发了聚丙烯酸钠 (Sodium polyacrylate, PANa)结合纤维素的创新型凝胶电解 质, 通过将纤维素、聚丙烯腈与亚甲基双丙烯酰胺 (MBAA) 相互交联, 使得其形成的网络多孔、溶氧 量大、㓞性好。随着PANa的加入, 该凝胶电解质 的电导率从 $0.15 \mathrm{~S} \cdot \mathrm{cm}^{-1}$ 增加到 $0.28 \mathrm{~S} \cdot \mathrm{cm}^{-1}$ 。同时加 入 $2.7 \%$ 的纤维素, 使得电解质的断裂强度以及拉 伸强度得到了极大的提高, 能够拉伸 $1000 \%$ 。而仅 


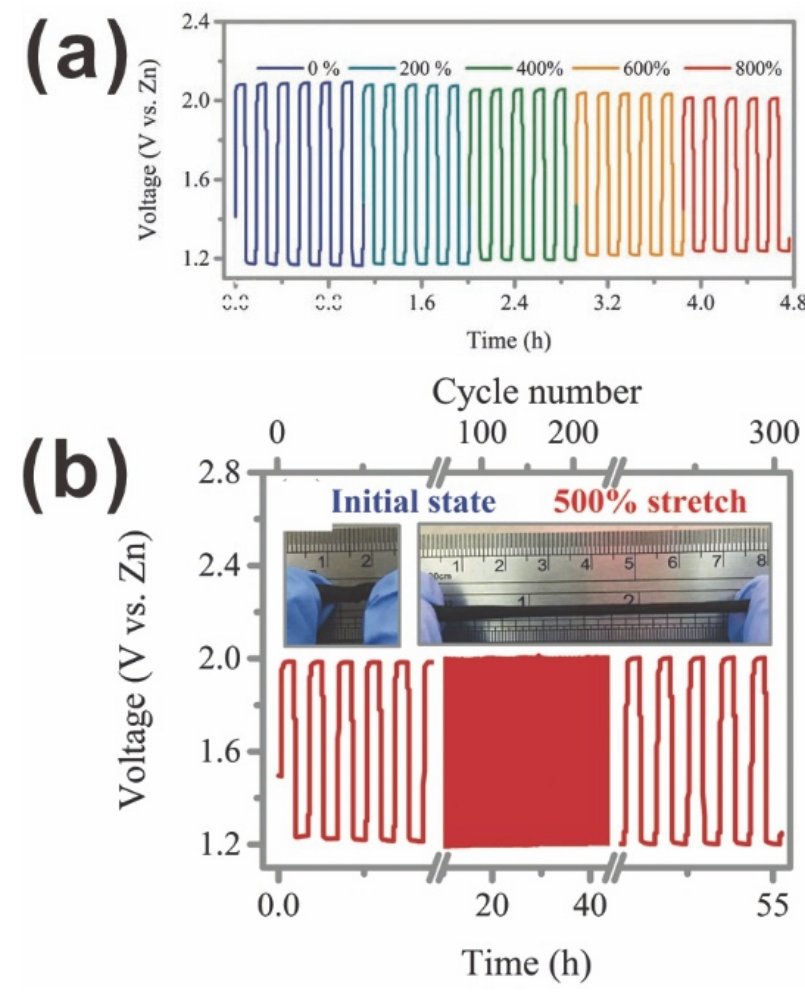

图 6 三明治与电缆构型锌-空气电池拉伸能力测试 16

Fig. 6 Tensile ability test of sandwich and cable type zinc-air batteries ${ }^{16}$.

(a) Galvanostatic discharge-charge cycling curves at a current density of $5 \mathrm{~mA} \cdot \mathrm{cm}^{-2}$; (b) Cycling test of fiber-shaped zinc-air

battery for rechargeability at a current density of $5 \mathrm{~mA} \cdot \mathrm{cm}^{-2}$ under $500 \%$ strain. The inset is the photographs of the fiber-shaped zinc-air battery at a fully released state and $500 \%$ strain. Adapted with the permission from Ref. 16. Copyright 1999-2021, John Wiley \& Sons, Inc.

仅使用PANa的电解质，拉伸强度仅为 $300 \%$ 。在碱 性溶液中如PAA、PAM和PANa，水凝胶网络极容 易破坏，这是由于 $\mathrm{OH}^{-}$易与水凝胶中的 $-\mathrm{COOH}$ 或一 $\mathrm{NH}_{2}$ 发生反应。通常解决的办法是加入 $\mathrm{NaOH}$ 作为中和剂, 但又无可避免的增加了体积, 并减少 了凝胶电解质的拉伸强度。但是, 通过加入纤维素 形成的纤维素链可以有效的抑制水凝胶的溶胀度 增加, 明显改善机械性能。因此, Ma等制成的三 明治与纤维状锌-空电池, 拉伸性分别达到了 $800 \%$ 与 $500 \%$, 并且在进行拉伸后, 电池的充放电循环 性能没有发生明显的变化(图6a, b)。

此外, 研究表明, 共价体系的共价交联聚合物 网络能确保水凝胶有足够的硬度和韧性。同时, 非 共价交联键在加载过程中耗散能量, 提高了双网 络水凝胶的抗疲劳性能和恢复能力。如, Sun等 ${ }^{17}$ 研发了碱性双网络的纤维素类凝胶电解质, 聚(2丙烯酰胺-2-甲基丙磺酸钾盐) (PAMPS-K)和互穿
甲基纤维素(MC)相结合起来, 形成的水凝胶即便 是在 $-20^{\circ} \mathrm{C}$ 下也能保持相当好的机械强度以及离 子导电性。在 $25^{\circ} \mathrm{C}, 5 \mathrm{~mol} \cdot \mathrm{L}^{-1} \mathrm{KOH}$ 情况下, 离子 电导率达到 $105 \mathrm{mS} \cdot \mathrm{cm}^{-1}$ 。组装成的凝胶型锌-空气 电池比容量密度达到了764.7 $\mathrm{mAh} \cdot \mathrm{g}^{-1}$, 能量密度 达到了 $850.2 \mathrm{mWh} \cdot \mathrm{g}^{-1}$ 。

\section{3 氧化石墨烯类凝胶电解质}

氧化石墨烯被证明有高离子电导率以及化学 稳定性, 同时含有大量含氧基团的电子绝缘体(环 氧基、羟基和羧基), 可轻易功能化以提高其离子 电导率 ${ }^{18,19}$ 。因此, Zhang等 ${ }^{20}$ 将纤维素与氧化石墨 烯先进行了季铵 (QA)功能化处理, 随后层层堆 叠, 以及离子交换处理。处理后的氧化石墨烯与纤 维素包含大量的 $\mathrm{OH}^{-}$离子(图7a), 使得电解质的电 导率进一步提升 $\left(58.8 \mathrm{mS} \cdot \mathrm{cm}^{-1}, 70^{\circ} \mathrm{C}\right)$, 而普通的 氧化石墨烯只有 $2.5 \mathrm{mS} \cdot \mathrm{cm}^{-1}$ 。图 $7 \mathrm{~b}$ 展示了 $\mathrm{SEM}$ 下 观察到的季铵官能团功能化石墨烯(QAFCGO), 薄 膜截面十分均匀平整(图7c)。其扫描电镜图像(图 7d)和其上的插图(透射电镜图像)显示纤维素紧密 交织的网络结构。图7e也展现出该电解质良好的 拉伸性能。组装成锌-空气电池后, 它的稳定性以 及循环能力都要优于商用的A201。如图7f所示, QAFCGO的开路电压为 $1.41 \mathrm{~V}$, 与商用的A201相 似。电流密度超过 $20 \mathrm{~mA} \cdot \mathrm{cm}^{-2}$ 时, QAFCGO的电池 充放电的过电势要明显小于商用A201, 例如在60 $\mathrm{mA} \cdot \mathrm{cm}^{-2}$ 时 QAFCGO 的充电电势比 A201 小 291 $\mathrm{mV}$, 放电电势比商用的 $\mathrm{A} 201$ 小 $154 \mathrm{mV}$ 。在电流密 度为 $1 \mathrm{~mA} \cdot \mathrm{cm}^{-2}$ 时, $\mathrm{QAFCGO}$ 循环了 30 圈 $(10 \mathrm{~min}$ 放 电以及 $10 \mathrm{~min}$ 充电为一个循环), 而商用 $\mathrm{A} 201$ 仅仅 循环了 15 圈。

基于上述等人的工作, Song等 ${ }^{21}$ 将氧化石墨烯 与聚合物相结合开创了一个全新的方向。他们将 PVA、PAA、GO与KI相混合, 经过PVA等修饰后, 形成了交联的复合网络结构。由于氧化石墨烯表 面和PVA链上有丰富的氧官能团, PVA和GO通过 形成氢键进行交联。它的工作时长达到了 $200 \mathrm{~h}$, 对比PVA为主的锌-空气电池(放电时长 $3 \mathrm{~h}$ ), KIPVA为主的锌-空电池放电时长长达 $166 \mathrm{~h}$ 。同样, 采用 KI-PVA组装而成的柔性锌-空气电池具备很 好的弯折性与纺织性, 可为各种电子产品充电。

综上所述, 目前主流的电解质制备方式是将 聚合物、纤维素、氧化石墨烯等物质相结合, 来制 备高性能电解质。但是要同时满足机械性能、耐高 低温能力、高离子电导率等要求的电解质还未诞 生, 也亟待人们的探索。柔性锌-空气电池常用电 解质及其性能、特点总结如表1所示。当然, 除了 

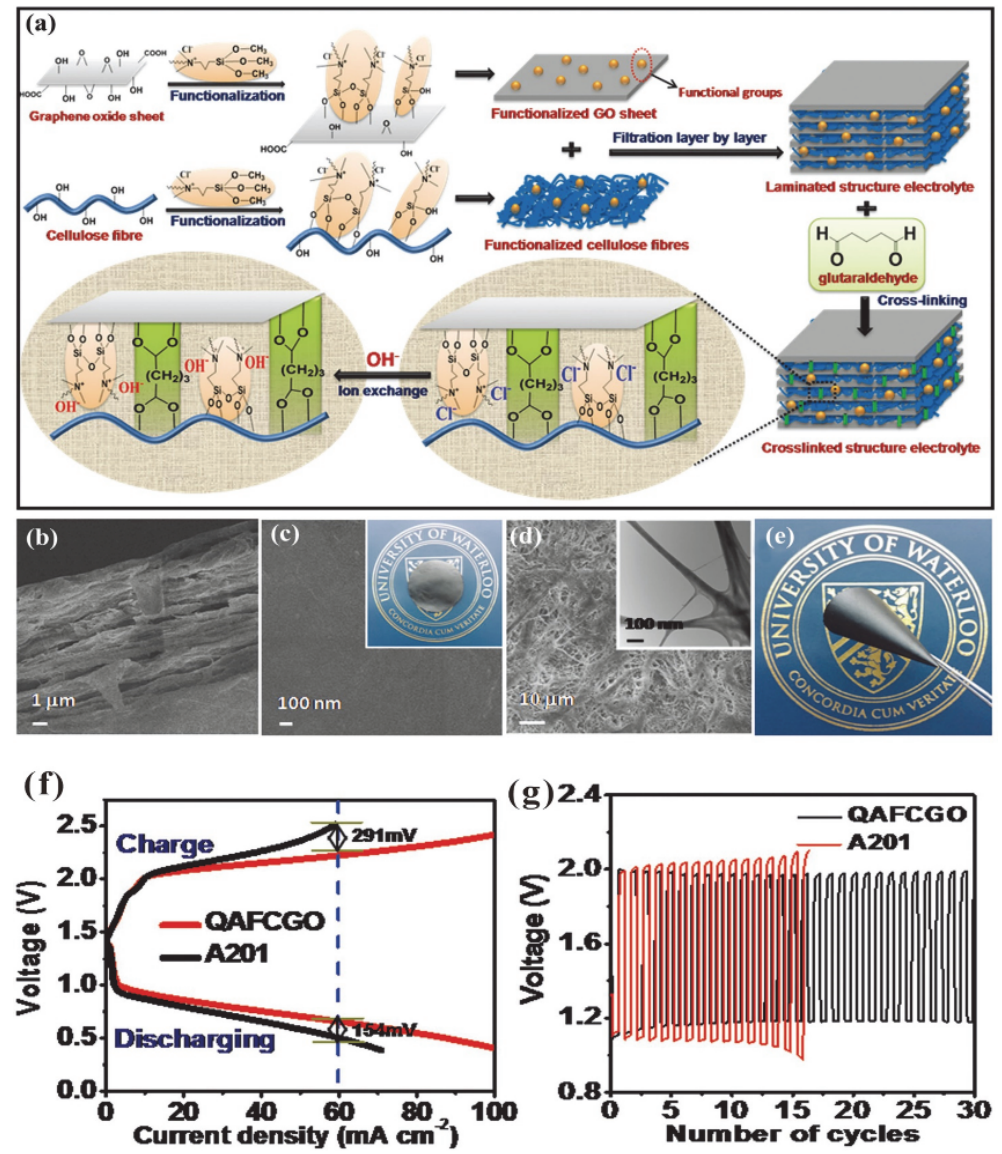

图 7 石墨烯与纤维素交联原理、微观结构电镜及性能测试图 20

Fig. 7 Cross-linking principle of graphene and cellulose, microstructure and comparison of charge-discharge polarization curves and cycles between QAFCGO and commercial A201 ${ }^{20}$.

(a) Schematic diagram of the overall preparation procedure for the QAFCGO membrane; (b) a SEM image (cross section) of the QAFCGO membrane;

(c) a SEM image (surface view) of the QAFCGO membrane with an inset photograph of QAFCGO membrane showing flat and uniform GO surface;

(d) a SEM image of the cellulose dense interwined network structure with an inset TEM image of cellulose fibers; (e) a photograph of the QAFCGO membrane showing flexibility; (f) charge and discharge polarization curves of the batteries using the QAFCGO and A201 membranes;

(g) galvanostatic charge and discharge cycling of the batteries using the QAFCGO and A201 membranes at $1 \mathrm{~mA} \cdot \mathrm{cm}^{-2}$ with a 20 min per cycle period. Adapted with the permission from Ref. 20. Copyright 1999-2021, John Wiley \& Sons, Inc.

要考虑电解质本身的性能外, 还要考虑组件与组 件之间的联系, 特别是凝胶电解质与空气阴极、锌 阳极的界面问题。下面将分别介绍凝胶电解质与 空气阴极、锌阳极之间的界面问题。

\section{4 凝胶电解质与阴阳极之间的界面问题}

\section{1 凝胶电解质与空气阴极界面问题}

聚合物电解质的锌-空气电池与传统液体锌空气电池一样, 反应过程也取决于三相反应界面。 不同的是电解液是存蓄在聚合物电解质中，从而 可以减少电解液的挥发和碱爬现象的发生, 延长 电池的寿命。但同时存在的挑战就是电解质与锌 片和空气阴极的接触并不能像电解液一样充分的 浸润阳极与阴极, 因此使得反应发生过程较慢, 甚
至严重影响性能。电解液不能够充分润湿空气阴 极, $\mathrm{OH}^{-}$的移动速率与范围就会受到很大的限制。 为此, 提高 $\mathrm{OH}^{-}$浓度, 以及制备具有微孔结构、高 机械强度的凝胶电解质就相当必要。如上文提到 的Zhang 等 ${ }^{20}$ 研发的季铵官能团优化后的氧化石墨 烯与纤维素电解质。与此同时, 改善空气阴极的基 底，使其具有高浸润性，提高 $\mathrm{OH}^{-}$在界面之间的流 动性亦或是改变 $\mathrm{OH}^{-}$的反应途径, 也是一个很好的 决策。例如, Haynes等研究了 $\mathrm{KI}$ 对 $\mathrm{OH}^{-}$的影响 22 。

无KI添加剂:

阴极: $4 \mathrm{OH}^{-} \rightarrow \mathrm{O}_{2}+2 \mathrm{H}_{2} \mathrm{O}+4 \mathrm{e}^{-}$

有KI添加剂:

阴极: $\mathrm{I}^{-}+6 \mathrm{OH}^{-} \rightarrow \mathrm{IO}_{3}{ }^{-}+3 \mathrm{H}_{2} \mathrm{O}+6 \mathrm{e}^{-}$

它改变了 $\mathrm{OER}$ 的反应途径, 同时 $\mathrm{IO}_{3}^{-}$与 $\mathrm{I}^{-}$反应 
表 1 柔性锌-空气电池常用电解质及其性能、特点

Table 1 Typical GPEs used in flexible ZABs.

\begin{tabular}{|c|c|c|c|c|c|}
\hline Gel Electrolyte & Additive & Ionic Conductivity & Characteristic & Weakness & Reference \\
\hline PVA & & $36.1 \mathrm{mS} \cdot \mathrm{cm}^{-1}$ & Bendable and stretchable & Low conductivity & {$[12]$} \\
\hline Porous PVA & & $38.3 \mathrm{mS} \cdot \mathrm{cm}^{-1}$ & High conductivity & & {$[12]$} \\
\hline Porous PVA & $\mathrm{SiO}_{2}$ & $57.3 \mathrm{mS} \cdot \mathrm{cm}^{-1}$ & High cycle-life & & {$[12]$} \\
\hline PEO-PPO-PEO & & $29 \mathrm{mS} \cdot \mathrm{cm}^{-1}$ & Excellent $\mathrm{CO}_{2}$-tolerance & Low conductivity & {$[13]$} \\
\hline PANa & Cellulose/MBAA & $280 \mathrm{mS} \cdot \mathrm{cm}^{-1}$ & High conductivity & $\begin{array}{l}\text { Complicate } \\
\text { fabrication }\end{array}$ & [16] \\
\hline PVA-PEO & Glass fibers & $10 \mathrm{mS} \cdot \mathrm{cm}^{-1}$ & Strong mechanical properties & low conductivity & [7] \\
\hline GO & & $2.5 \mathrm{mS} \cdot \mathrm{cm}^{-1}$ & & & {$[20]$} \\
\hline QAFCGO & Cellulose & $58.8 \mathrm{mS} \cdot \mathrm{cm}^{-1}$ & $\begin{array}{l}\text { High conductivity and } \\
\text { stretchability }\end{array}$ & High cost & {$[20]$} \\
\hline PVA-PAA-GO & KI & $155 \mathrm{mS} \cdot \mathrm{cm}^{-1}$ & Excellent stability & $\begin{array}{l}\text { Complicate } \\
\text { fabrication }\end{array}$ & {$[21]$} \\
\hline $\mathrm{PAM} / \mathrm{PAA}$ & Gly/APS & $26 \Omega$ & $\begin{array}{c}\text { High- and low-temperature } \\
\text { tolerant }\end{array}$ & $\begin{array}{c}\text { Low conductivity } \\
\text { at RT }\end{array}$ & {$[15]$} \\
\hline
\end{tabular}

的电势 $\left(0.26 \mathrm{~V} v s\right.$. SHE) 要远远小于 $\mathrm{O}_{2}$ 与 $\mathrm{OH}^{-}$的电 势 $(0.401 \mathrm{~V} v s$. SHE), 从根本上降低了基于 $\mathrm{KI}$ 的锌空气电池所需要的充电电压。

除了上述问题外, 凝胶电解质与空气电极之 间的界面稳定性容易在弯折的情况下被破坏, 这 需要使得电解质与空气电极同时具备良好的机械 性能与贴合性 ${ }^{23}$ 。

\section{2 凝胶电解质与锌阳极界面问题}

对于锌-空气电池来说, 枝晶产生会导致金属 阳极的断裂, 降低电池的性能 23 。情况更糟的是, 枝晶会刺破隔膜, 导致电池短路 ${ }^{1}$ 。与此同时, 碳 酸盐沉积的问题会导致锌-空气电池的循环能力 降低, 缩短电池的使用寿命 ${ }^{24}$ 。枝晶通常是由于锌 酸盐离子不均匀沉积导致的, 增大电解质与金属 电极之间的接触面积有助于缓解这一问题。例如, $\mathrm{Xu}$ 等 ${ }^{7}$ 从传统的锌箔改用锌弹簧, 加大了界面之间 的接触。与此同时, Banik等 ${ }^{25}$ 发现用聚乙二醇 (PEG)可以抑制锌枝晶的生长, 随着PEG浓度的提 高有效降低了锌枝晶的含量。他们又发现通过增 加聚乙烯亚胺(Polyethyleneimine, PEI)的含量, 也 有效的抑制了枝晶的生长。Khezri等 ${ }^{26}$ 认为当过硫 酸钾(KPS)加入到碱性溶液中, 它通过 $\mathrm{O}-\mathrm{O}$ 键的 对称断裂进行热分解, 形成一个由氢离子催化的 一级反应。产生的硫酸盐自由基进一步诱导氧化。 因此, KPS可以加速 $\mathrm{OH}^{-}$离子的催化氧化并相应地 改善充电过程。加入 $0.045 \% \mathrm{KPS}$ 能够有效地增加 锌-空气电池的循环能力。

在考虑完界面设计之后, 锌阳极与空气阴极 本身的柔性程度也成为制约柔性锌-空气电池的
关键, 下面将分别进行论述。

\section{5 金属电极的优化}

通常人们用金属锌来作为锌-空气电池的负 极材料, 为了制备柔性的锌-空气电池, 对锌阳极 进行修饰也很有必要, 使其具备一定的机械强度 和韧性。本节将讨论目前对柔性锌阳极的修饰方 式, 以及未来的发展趋势。

\section{1 电镀锌阳极}

$\mathrm{Qu}$ 等 ${ }^{27}$ 将多孔锌阳极电镀到铜泡沫上, 既发 挥了三维多孔锌的电流收集能力, 又可以与集流 体铜泡沫紧密结合, 如图8a所示。同时他们用PVA$\mathrm{TEAOH}$ (氢氧化四乙铵)-KOH作为电解质, 与常用 的PVA $+\mathrm{KOH}$ 相比大大增强了保水能力。制备出 的柔性锌-空气电池可以在弯折 $0^{\circ} 、 45^{\circ} 、 90^{\circ}$ 后, 充 放电循环曲线没有明显的变化(图8b), 同时三个串 联的锌-空气电池在弯折后也能为LED灯供电(图 $8 \mathrm{c}$ )。此外, $\mathrm{Pan}$ 等 ${ }^{28}$ 采用三聚氭胺海绵作为碳源, 热解后将 $\mathrm{Fe}-\mathrm{Co}_{3} \mathrm{O}_{4}$ 的纳米阵列电镀在上面, 同时 将锌纳米阵列电沉积到另一块海绵碳上, 两者结 合出的三明治固态电池具有惊人的可压缩性(图 9a)。同时测试出电池在不同程度下按压后, 充放 电循环没有任何变化, 展现出极大的抗压性(图 $9 b)$ 。虽然Pan等的锌-空气电池具有较强的按压性 能, 但是弯折与拉伸能力还有待提高。为进一步提 高锌阳极机械强度, $\mathrm{Qu}$ 等 ${ }^{29}$ 又用聚氨酯海绵 (PUS), 将其与Exoflex薄膜相结合, 进而浸泡在氯 化锡与氯化钯的溶液中, 使其具有表面活性与硫 酸铜发生反应(镀铜), 使海绵具有导电能力。随后 

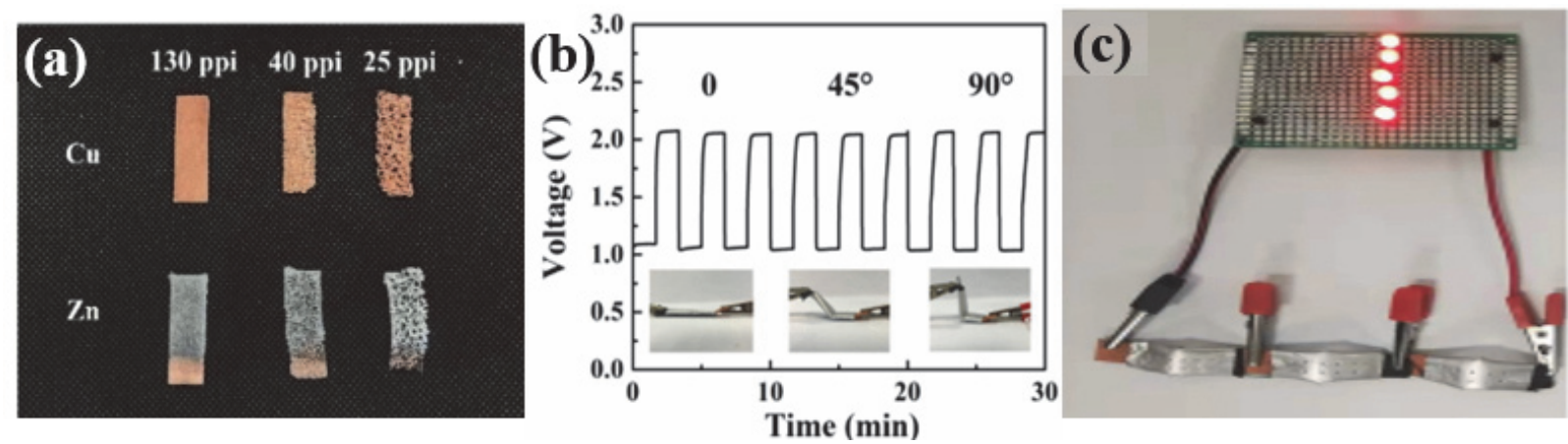

图 8 商用泡沫铜及不同密度锌覆盖铜的照片和组装柔性锌-空电池机械性能测试 27

Fig. 8 Photographs of commercial copper foams and zinc coverings and mechanical properties testing of assembled flexible zinc-air batteries ${ }^{27}$.

(a) Photographs of the commercial $\mathrm{Cu}$ foams and the $\mathrm{Zn}$-coated foams with 130, 40, and 25 ppi, respectively; (b) galvanostatic charge/discharge cycling tests of the ZABs with each cycle being $200 \mathrm{~s}$ tested under $0^{\circ}, 45^{\circ}$ and $90^{\circ}$ bending conditions; (c) photographs of five red LEDs in parallel powered by the three as-prepared ZABs in series under bending state, respectively. Adapted with the permission from Ref. 27. Copyright 1999-2021, John Wiley \& Sons, Inc.

(a)
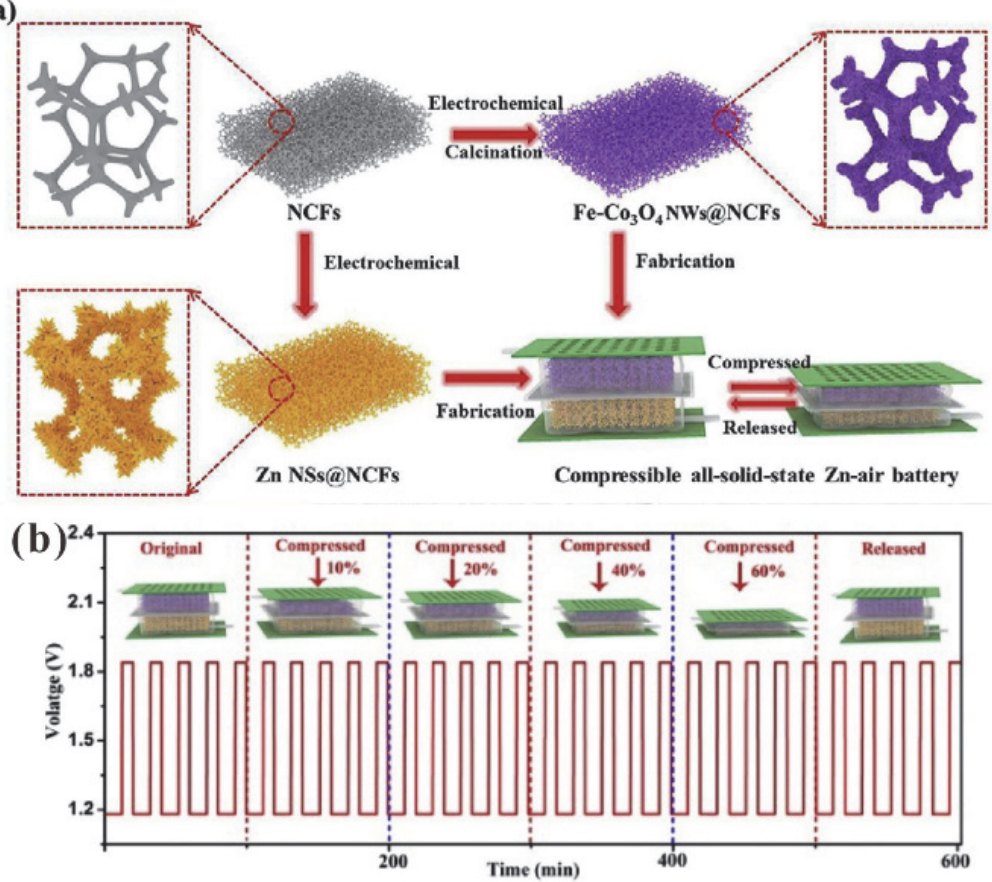

图 9 可挤压和可充电的锌-空气电池制造工艺示意图以及机械性能测试图 28

Fig. 9 Manufacturing process photographs and mechanical test of the extrudable and rechargeable all solid state ZAB ${ }^{28}$.

(a) Schematic illustration of the fabrication processes for the squeezable and rechargeable all-solid-state ZAB; (b) galvanostatic discharging/charging cycling curves. Adapted with the permission from Ref. 28. Copyright 2021, Elsevier B.V.

再将锌电沉积到海绵上, 制备出柔性的锌阳极。他 们用PUS@Zn,PAA水凝胶电解质以及PUS@Ni三 者组合成全固态柔性锌-空气电池, 其可以拉伸 $100 \%$ ，也可以扭曲 $160 \%$ ，同时有非常强的抗疲劳 恢复能力。在进行几百次的弯折和扭曲后仍能恢 复成原有的形状, 而普通的锌板、镍泡沫则已经不 能恢复到原来的形状。

将锌与其他材料复合, 既可以发挥其他材料 的优势, 也不会破坏锌-空气电池的运作机理, 甚
至能起到相应的协同作用。与此同时, 改变锌阳极 本身的机械性能也至关重要。

\section{2 锌阳极的机械性能改善}

$\mathrm{Xu}$ 等 ${ }^{7}$ 将锌线绕着铜棒缠绕成锌弹簧, 使其具 有很强的拉伸能力。随后将做好的凝胶电解质涂 敷在锌阳极上, 待凝固后滴涂 $\mathrm{RuO}_{2}$, 最后在聚四 氟乙烯板上用CNT包覆, 成功做成一维纤维状锌空气电池(图10a)。对其进行机械性能测试, 表现 出不错的拉伸 $(10 \%)$ 与弯折能力(图10b，c)。目前 
对于锌阳极的优化仅限于锌的弹簧化, 或直接用 锌线代替, 主流的改善方式仍然是将锌与其他材 料复合。例如Mitra等 ${ }^{30}$ 将锌粉、聚合物粘合剂、氧 化铋和纯化的多壁碳纳米管(MWCNTs)混合形成 阳极, $\mathrm{Fu}$ 等 ${ }^{3}$ 将锌粉与碳纳米纤维和炭黑进行结 合, 都展现出极大的柔韧性, 期望未来更多锌阳极 的优化方式的出现。

\section{6 柔性空气阴极}

空气阴极作为锌-空气电池中 ORR与 OER反 应发生的场所, 对电池的性能起到至关重要的作 用。ORR反应发生在三相界面处, 亲水性通道运输 电解液, 而相对应的疏水性微孔则负责氧气的扩 散以及防止电解液的泄露, 这就需要空气阴极疏

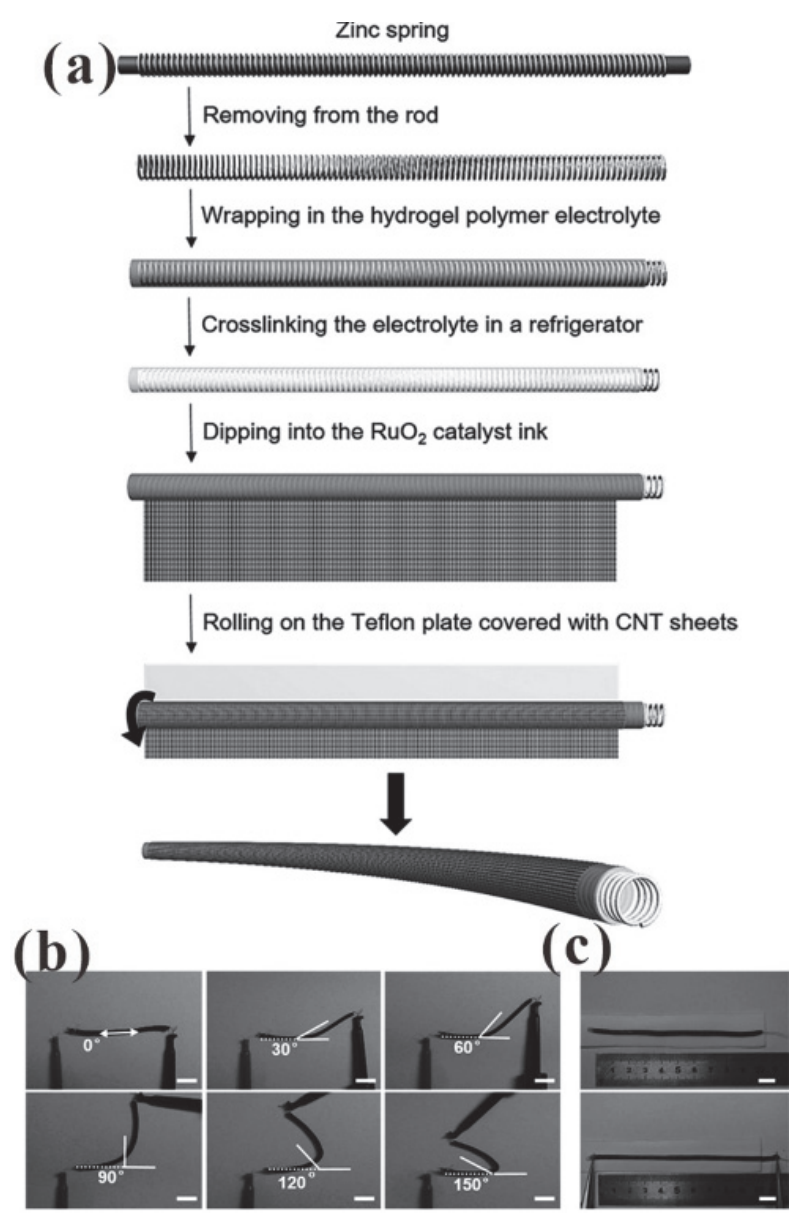

图 10 锌弹簧制备锌-空气电池流程以及机械性能测试 7

Fig. 10 Zinc spring preparation process for ZABs and mechanical properties testing ${ }^{7}$.

(a)Schematic illustration to the fabrication of the fiber-shaped $\mathrm{Zn}$-air battery; (b) photographs of a fiber-shaped $\mathrm{Zn}$-air battery bent to increase angles. scale bar: $1 \mathrm{~cm}$; (c) photographs of a fiber-shaped $\mathrm{Zn}$-air battery before and after stretching by $10 \%$, scale bar: $1 \mathrm{~cm}$. Adapted with the permission from Ref. 7. Copyright 2021, Elsevier B.V.
水性与亲水性要保持一定的平衡。同时, 空气阴极 中的基底一方面要起到承担载体并导电的作用, 另 一方面也要让气体通过, 因此选择良好的基底至 关重要。接下来这一部分将会介绍柔性锌-空气电 池中空气阴极常用的基底。

由于碳布具有良好的弯折能力, 因此被很多 人选择作为柔性基底。例如, Guan等 32 就以碳布作 为基底, 在碳布上原位生长氮与碳共掺的氧化钴 $\left(\mathrm{NC}-\mathrm{Co}_{3} \mathrm{O}_{4}\right)$ (图11a)。SEM下可以观察到 $\mathrm{NC}-\mathrm{Co}_{3} \mathrm{O}_{4}$ 均匀分布于碳布的表面(图11b，c)。他们以此组装 出的柔性锌-空气电池具有高开路电压 $(1.44 \mathrm{~V})$, 高 容量(387.2 $\left.\mathrm{mAh} \cdot \mathrm{g}^{-1}\right)$, 优秀的循环能力(60圈)的特 点。除了以碳布作为基底, $\mathrm{Liu}^{\text {等 }}{ }^{33}$ 以自制的碳纳米 膜(CNMF) 作为基底, 做出的柔性锌-空气电池开路 电压为 $1.40 \mathrm{~V}$, 与此同时最大功率密度也达到了 $26.5 \mathrm{~mW} \cdot \mathrm{cm}^{-2}$ 。

虽然碳材料具备高导电性、低成本的显著优 势, 但在锌-空气电池充电过程中, 高氧化电位很 容易使得碳被腐蚀, 最终会降低锌-空气电池的性 能。因此为解决碳腐蚀所带来的影响, 人们通常是 选择高度石墨化的碳, 例如CNT或者石墨烯作为 基底。另一种方式就是用金属来作为替代, 例如镍 网、不锈钢网、铜网、铜泡沫、镍泡沫等, 它们都 具备较好的柔韧性以及导电能力。例如, $\mathrm{Xu}$ 等 ${ }^{34}$ 用 镍网作为基底, 在上面进行催化剂的原位生长。 $(\mathrm{Ni}, \mathrm{Co})_{3} \mathrm{O}_{4}$ 纳米片直接附着在镍网上并且不需要 粘结剂, 制作出的柔性锌-空气电池具有较好的柔 性以及出色的电化学稳定性, 结构如图12a。如图 $12 \mathrm{~b}$ 所示, 它的最大功率密度达到了 $74 \mathrm{~mW} \cdot \mathrm{cm}^{-2}$ (商用 $\mathrm{Pt} / \mathrm{C}$ 只有 $41 \mathrm{~mW} \cdot \mathrm{cm}^{-2}$ )。与此同时, 在恒流放 电的情况下, 可以在不同弯折程度下稳定放电 $45 \mathrm{~h}$ (图12c)。Xu等又对其进行机械性能的测试, 在弯 折 $60^{\circ}$ 与 $120^{\circ}$ 后电池电压仍保持稳定(图 $12 \mathrm{~d}$ )。不锈 钢网也是一个不错的选择, 例如, $\mathrm{Wu}$ 等 ${ }^{35}$ 水热的 方式在不锈钢上生长 $\mathrm{Co}_{3} \mathrm{O}_{4}$, 同时在镍网上生长钴 镍氧化物 $\left(\mathrm{Co}_{3-x} \mathrm{Ni}_{x} \mathrm{O}_{4}\right)$ 的纳米线, 最后通过层压的 方式将两层整合到一起, 制作出无需粘结剂高灵 活性的空气阴极。该电池充分利用了三明治构型 的优势, 高的循环性使电池工作 $600 \mathrm{~h}$ 而过电势只 有轻微的增加。

除了以上基底外，碳材料与金属材料复合作 为空气阴极基底也是一个不错的方向, 亟待人们 探索。

\section{7 柔性锌-空气电池的构型}

对于柔性锌-空气电池来说, 在各种外力作用 


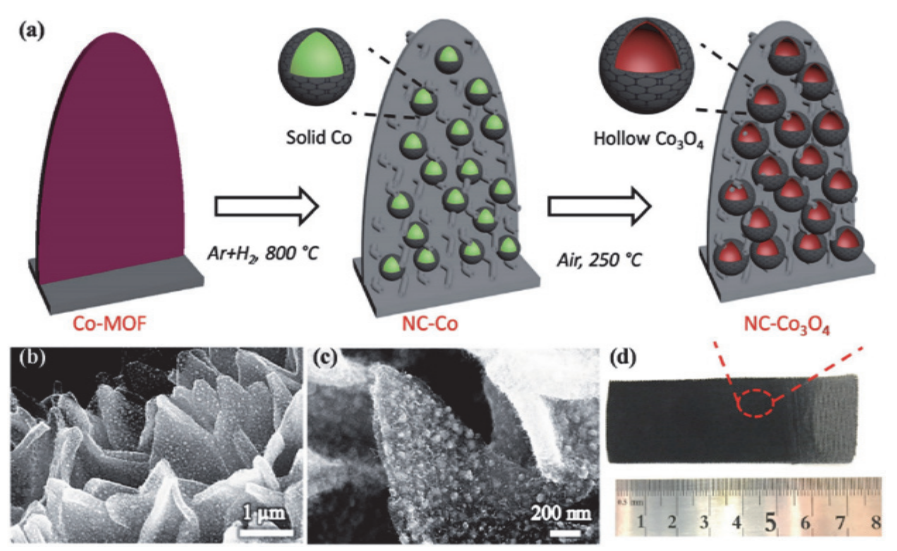

图 11 NC-C $0_{3} \mathrm{O}_{4}$ 制备工艺以及 $\mathrm{SEM}$ 表征图 ${ }^{32}$

Fig. 11 Preparation process of $\mathrm{NC}-\mathrm{Co}_{3} \mathrm{O}_{4}$ and $\mathrm{SEM}$ characterization ${ }^{32}$.

(a) Schematic illustration of the fabrication process for hierarchical NC- $\mathrm{Co}_{3} \mathrm{O}_{4}$ arrays on flexible carbon cloth; (b) and (c) SEM and (d) digital images of NC- $\mathrm{Co}_{3} \mathrm{O}_{4}$ nanoarrays on carbon cloth. Adapted with the permission from Ref. 32. Copyright 1999-2021, John Wiley \& Sons, Inc.

(a)

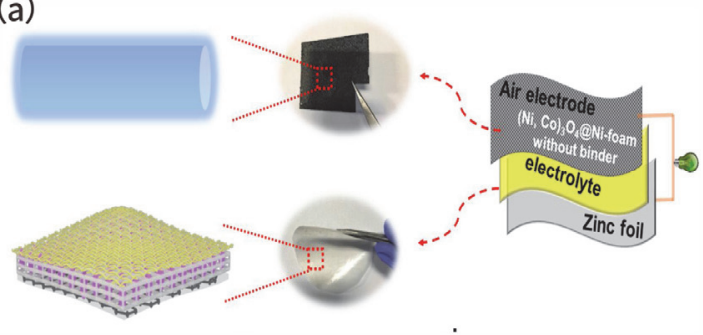

(c)

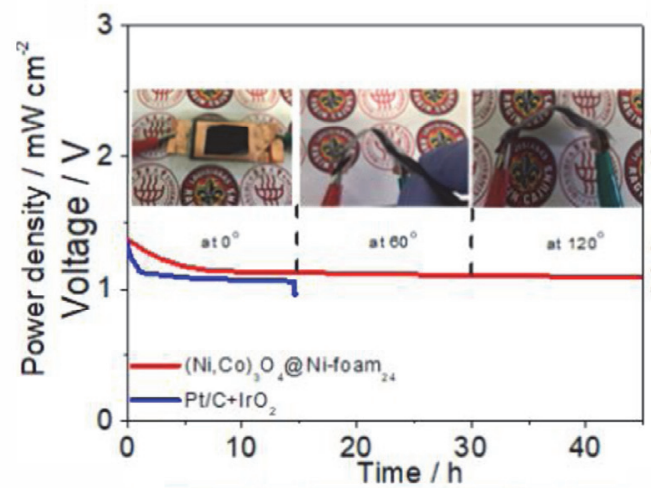

(b)

(d)
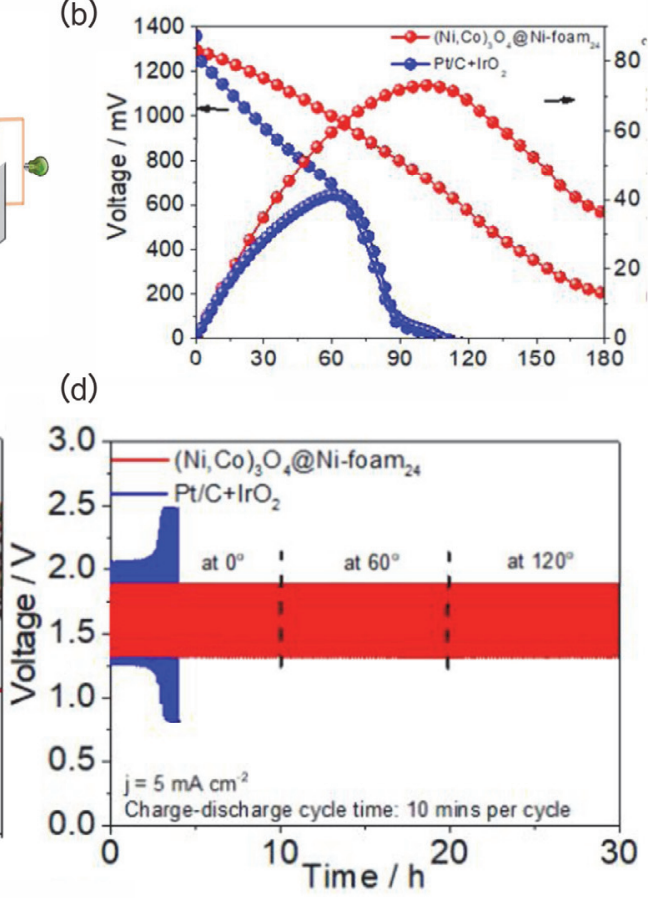

图 $12(\mathrm{Ni}, \mathrm{Co})_{3} \mathrm{O}_{4} @ \mathrm{Ni}$-foam 制备原理以及电化学性能机械性能测试 ${ }^{34}$

Fig. 12 Preparation principle and electrochemical properties and mechanical properties testing ${ }^{34}$.

(a) Schematic diagram of the flexible zinc-air battery. $\mathrm{Pt} / \mathrm{C}+\mathrm{IrO}_{2}$ and $(\mathrm{Ni}, \mathrm{Co})_{3} \mathrm{O}_{4} @ \mathrm{Ni}$-foam; (b) the polarization curve and corresponding power density plot; (c) the long-time galvanostatic discharge curves under different bending conditions at $5 \mathrm{~mA} \cdot \mathrm{cm}^{-2}$; (d) the $\mathrm{C}-\mathrm{D}$ cycling curves.

Adapted with the permission from Ref. 34. Copyright 2021, Elsevier B. V.

下仍能保持稳定的电化学性能是相当重要的。同 样重要的是, 在各种实际应用当中, 柔性锌-空气 电池除了能够拉伸、弯折、扭曲之外, 仍能保持记 忆弹性, 恢复原来的形变量也是很重要的。接下来 将会讨论, 柔性锌-空气电池最常见的两种构型, 三明治和电缆式构型, 以及未来的发展方向。

\section{1 三明治构型}

传统的三明治结构的锌-空气电池构型如图
13所示。Yang等 ${ }^{36}$ 将催化剂负载于碳布上, 电解液 与隔膜用PVA- $\mathrm{KOH}$ 水凝胶电解质, 最后一层用锌 板作为金属阳极, 三层整合到一块, 制备出三明治 构型电池。组装的电池表现出优异的稳定性, 电池 经过 670 个充放电循环后 $(112 \mathrm{~h})$, 电压差仅仅从 $1.14 \mathrm{~V}$ 增加到 $1.38 \mathrm{~V}$ 。在电池机械性能上, 也表现 出很好的弯折性能。在分别弯折了 $0^{\circ} 、 90^{\circ} 、 180^{\circ}$ 之后, 电池电压差变化不大。电池功率密度达到了 


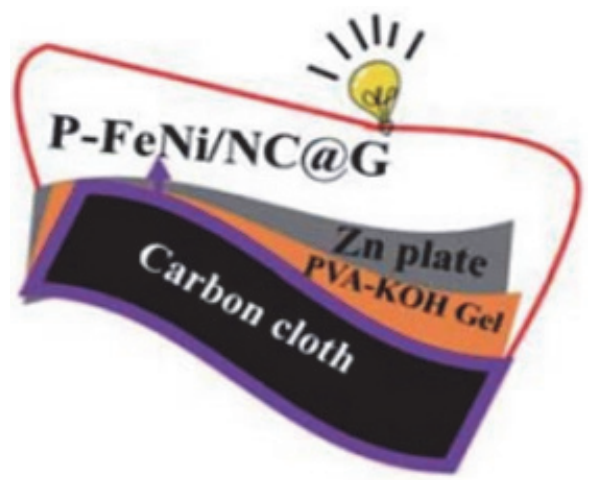

图 13 三明治结构锌-空气电池 ${ }^{36}$

Fig. 13 Sandwich structure of a zinc-air battery ${ }^{36}$. Adapted with the permission from Ref. 36. Copyright 2020, the Royal

Society of Chemistry.

$87.7 \mathrm{~mW} \cdot \mathrm{cm}^{-2}$, 远高于商用 $\mathrm{Pt} / \mathrm{C}+\mathrm{RuO}_{2}(51.6$ $\left.\mathrm{mW} \cdot \mathrm{cm}^{-2}\right)$ 的功率密度。 Tan等将钴与氧化钴 $(\mathrm{Co}-$ $\mathrm{CoO}){ }^{37}$ 的颗粒包裹进 N、S 掺杂的碳中 (记为 $\mathrm{Co}-$ $\mathrm{CoO} @ \mathrm{NSC})$, 并且成功应用于三明治构型的锌-空 气电池上。该电池开路电压达到 $1.46 \mathrm{~V}$, 比容量在
电流密度为 $10 \mathrm{~mA} \cdot \mathrm{cm}^{-2}$ 下达到了 $759 \mathrm{mAh} \cdot \mathrm{g}^{-1}$, 电 流密度为 $20 \mathrm{~mA} \cdot \mathrm{cm}^{-2}$ 时达到了 $678 \mathrm{mAh} \cdot \mathrm{g}^{-1}$, 相对 应的能量密度分别为 $971 \mathrm{Wh} \cdot \mathrm{kg}^{-1}$ 以及 813 $\mathrm{Wh} \cdot \mathrm{kg}^{-1}$ 。 $\mathrm{Zhao}$ 等 ${ }^{38}$ 用 $\mathrm{CoFe} / \mathrm{Co}$ 的纳米颗粒嵌入到 Co-N共掺的碳上, 制备出具有双功能的催化剂。 他们同时制备的三明治构型柔性锌-空气电池在 弯折了各种角度下, 开路电压仍能保持 $1.46 \mathrm{~V}$ 。类 似的, Zhang等 ${ }^{39}$ 用 $\mathrm{CoNi}$ 合金掺杂的碳纳米片制备 的三明治构型锌-空气电池弯折不同角度之后也 能循环 $800 \mathrm{~min}$ 。

空气阴极通常由基底、催化剂、气体扩散层组 成, 过于笨重的结构会导致质量能量密度下降并 严重影响其大规模的应用。甚至在电池进行循环 的过程中，其中任何一部分的脱落会导致电池性 能遭到严重的破坏，因此独立自支撑的空气阴极 的设计与制备就显得尤为重要。Liu等 ${ }^{33}$ 制备出含 氮的钴与碳纳米管生长在碳纳米巨膜上 $(\mathrm{Co} / \mathrm{N} @ \mathrm{CNTs} @ \mathrm{CNMF})$ 的独立自支撑空气阴极, 他 们优化了空气电极的结构, 电池开路电压达到了

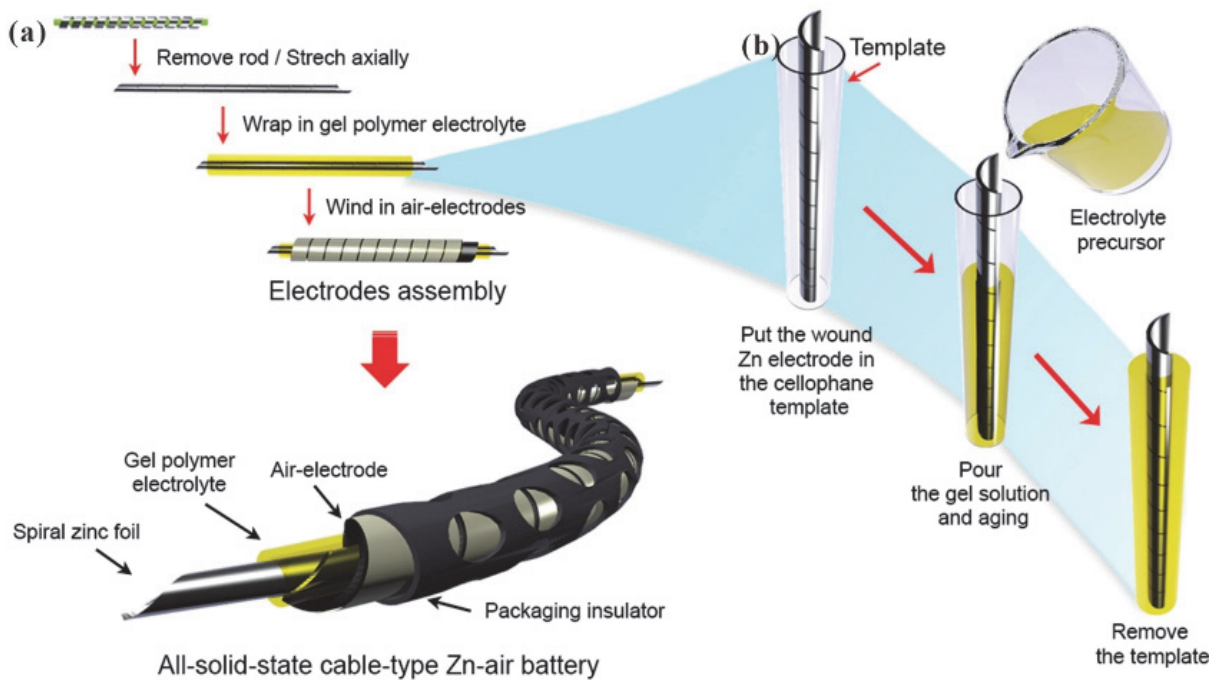

(c)

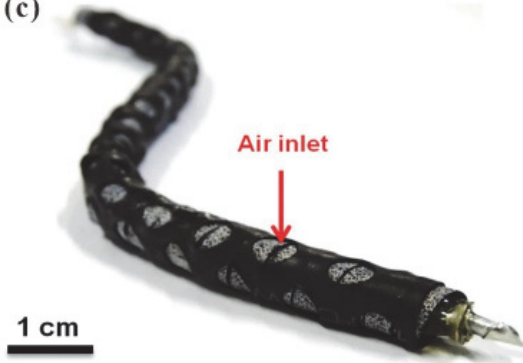

(d)

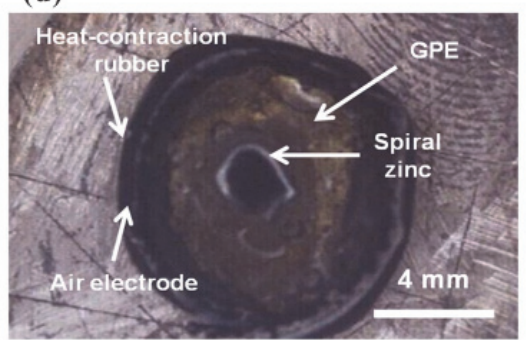

图 14 全固态电缆式锌-空气电池制造工艺、截面图及机械性能测试 40

Fig. 14 Manufacturing process, cross section and mechanical properties testing of all solid state cable zinc-air battery ${ }^{40}$.

(a) Schematic diagram of the all-solid-state cable-type flexible $\mathrm{Zn}$-air battery assembly and (b) coating process of gelatin-based gel polymer electrolyte (GGPE) and $0.1 \mathrm{~mol} \cdot \mathrm{L}^{-1} \mathrm{KOH}$ on the surrounding spiral zinc anode. Adapted with the permission from Ref. 40. Copyright 1999-2021, John Wiley \& Sons, Inc. 
$1.52 \mathrm{~V}$, 最大功率密度达到了 $133 \mathrm{~mW} \cdot \mathrm{cm}^{-2}$, 远高 于处于同样条件的 $\mathrm{Pt} / \mathrm{C}+\mathrm{RuO}_{2}$ 。与此同时 Co/N@CNTs@CNMF组装的柔性锌-空气电池功 率密度达到了 $26.5 \mathrm{~mW} \cdot \mathrm{cm}^{-2}$ 。机械性能方面, 表现 出很好的弯折能力, 在分别弯折了 $90^{\circ}$ 以及 $180^{\circ}$ 之 后充放电循环之间的电压差没有明显的变化。

对于三明治构型的柔性锌-空气电池, 人们大 多只对其进行简单的弯折、扭拧等测试, 而在实际 应用的过程当中也需要考虑很多因素, 如抗压力
测试、温度测试等。同时, 人们衡量柔性锌-空气 电池的标准也不一样, 有的采用各种角度的弯折 来测试电池的循环性能, 有的是反复弯折来衡量 电池的机械性能, 更为明确具体的标准尚未制定。 因此, 亟需引入一个形变量参数以及对应的电池 性能的参数, 来综合评估柔性电池的性能而不是 简单的描述各种柔性锌-空气电池各有所长。制定 一个良好的标准对柔性锌-空气电池未来的产业 化发展也大有裨益。
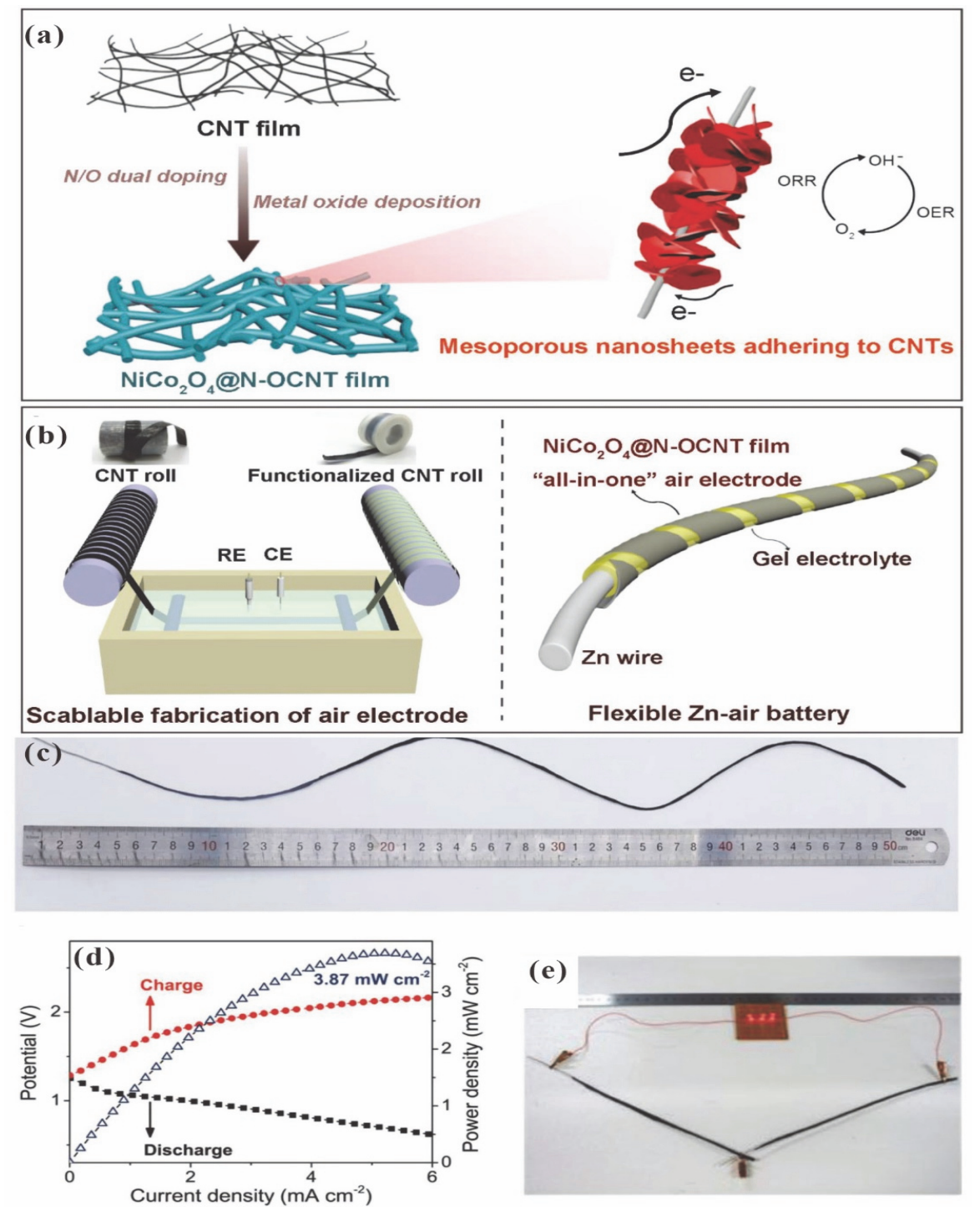

图 $15 \mathrm{NiCo}_{2} \mathrm{O}_{4} @ \mathrm{~N}-\mathrm{OCNT}$ 制备方式、电化学与机械性能测试 42

Fig. $15 \quad \mathrm{NiC0}_{2} \mathrm{O}_{4} @ \mathrm{~N}-\mathrm{OCNT}$ Preparation method, electrochemical and mechanical properties test ${ }^{42}$.

(a) Scheme showing the fabrication approach of $\mathrm{NiCo}_{2} \mathrm{O}_{4} @ \mathrm{~N}-\mathrm{OCNT}$ film; (b) scalable approaches of $\mathrm{NiCo}_{2} \mathrm{O}_{4} @ \mathrm{~N}-\mathrm{OCNT}$ air electrode (left) and the demonstration of flexible $\mathrm{Zn}$-air battery (right). $\mathrm{NiCo}_{2} \mathrm{O}_{4} @ \mathrm{~N}-\mathrm{OCNT}$ films all-in-one air electrode enabled high-performance flexible cable shaped

$\mathrm{Zn}$-air battery; (c) optical images of the cable-like flexible $\mathrm{Zn}$-air battery using the $\mathrm{NiCo}_{2} \mathrm{O}_{4} / \mathrm{N}$-OCNT composite film as the air electrode and zinc wire as the anode; (d) power density plots and charging-discharging polarization curves of Zn-air battery; (e) two 20-cm-long Zn-air batteries with straight, 


\section{2 电缆式构型}

相较于三明治构型柔性锌-空气电池, 电缆式 构型的柔性电池机械性能则更为突出。最初的电 缆式构型柔性锌-空气电池出现于2014年, 由Park 等 ${ }^{40}$ 提出。它的制造过程如图14a所示, 锌绕着钢 棒旋转制作成锌弹簧, 随后抽出钢棒, 并在锌上涂 上水凝胶的前驱体, 凝固后移除模板(图14b), 再 包覆空气电极, 最后用透气的热收缩管进行封装。

基于此, $\mathrm{Li}^{\text {等 }}{ }^{41}$ 以 $\mathrm{NiCo}_{2} \mathrm{O}_{4} @ \mathrm{NiCoFe}-\mathrm{OH}$ 作为 空气阴极, 将电缆式锌-空气电池的性能进一步完 善。得益于 $\mathrm{NiCO}_{2} \mathrm{O}_{4} @ \mathrm{NiCoFe}-\mathrm{OH}$ 优秀的双功能催 化性能, 在电流密度为 $50 \mathrm{~mA} \cdot \mathrm{cm}^{-2}$ 时, $\mathrm{NiCo}_{2} \mathrm{O}_{4} @ \mathrm{NiCoFe}-\mathrm{OH}$ 的充放电电势差为 $1.32 \mathrm{~V}$, 远小于 $\mathrm{NiCo}_{2} \mathrm{O}_{4}(1.61 \mathrm{~V})$ 以及商用 $\mathrm{Pt} / \mathrm{C}(1.58 \mathrm{~V})$ 。负 载有 $\mathrm{NiCo}_{2} \mathrm{O}_{4} @ \mathrm{NiCoFe}-\mathrm{OH}$ 催化剂的锌-空气电池, 其最大功率为 $55.2 \mathrm{~mW} \cdot \mathrm{cm}^{-2}$, 也明显大于 $\mathrm{NiCo}_{2} \mathrm{O}_{4}$ $\left(40.4 \mathrm{~mW} \cdot \mathrm{cm}^{-2}\right)$ 以及 $\mathrm{Pt} / \mathrm{C}\left(48.6 \mathrm{~mW} \cdot \mathrm{cm}^{-2}\right)$ 。在电流密 度为 $10 、 5 \mathrm{~mA} \cdot \mathrm{cm}^{-2}$ 时, 它的比容量分别达到了 723、740 $\mathrm{mAh} \cdot \mathrm{g}^{-1}$, 达到了锌-空气电池理论比容量 的 $90 \%$ 。 $\mathrm{NiCo}_{2} \mathrm{O}_{4} @ \mathrm{NiCoFe}-\mathrm{OH}$ 的能量密度高达 $864.2 \mathrm{Wh} \cdot \mathrm{g}^{-1}$ 。在电流密度为 $10 \mathrm{~mA} \cdot \mathrm{cm}^{-2}$ 时, 它的 过电势为 $0.83 \mathrm{~V}$, 远小于 $\mathrm{Pt} / \mathrm{C}$ 的 $1.23 \mathrm{~V}$ 。同时在进 行了 500 个充放电循环后 $(250 \mathrm{~h}, 30 \mathrm{~min}$ 每圈), 它 的电势差仍然保持稳定。在重复弯折了2000次之 后, 放电电压仅有轻微的下降, 证明了该电池具有 较高的机械稳定性。该电缆式锌-空气电池体积能 量密度高达 $38.1 \mathrm{mWh} \cdot \mathrm{cm}^{-3}$, 远高于同类型或三明 治型的锌-空气电池。

虽然人们将ORR与OER催化活性较高的催化 剂负载于电缆式锌-空气电池的空气阴极中, 但是 这些探索并没有优化空气电池的结构, 使它的电 化学性能进一步提高。若能采用优秀的结构设计, 可以使得电缆式的锌-空气电池有更广阔的市场 前景。通常使用的电缆式锌-空气电池, 三层结构 一圈一圈进行包覆, 使得单根锌-空气电池体积相 较于市场上采用的电池要粗大的多, 不可避免的 降低了其重量能量密度, 减小了使用的范围。为减 轻锌-空气电池的负担, Zeng等 ${ }^{42}$ 将催化剂、集流 体、基底整合到一起做出了一体式的全新电缆型 锌-空气电池。如图15a所示, 运用浮游催化沉积的 方式, 将催化剂与杂原子负载于 CNT上。同时如图 $15 \mathrm{~b}$ 所示, 将CNT进行可伸缩以及导电性能提高的 功能化处理。右图是制作出的一体式电缆型锌-空 气电池构型, 它具备纤细、体积较小的特点。该电 缆式锌-空气电池约有 $50 \mathrm{~cm}$ 长(图15c), 有着较好 的稳定性。同时在电流密度为 $0.25 \mathrm{~mA} \cdot \mathrm{cm}^{-2}$ 下, 放
电电位高达 $1.18 \mathrm{~V}$ (图15d)。该电缆式锌-空气电池 的最大功率密度为 $3.87 \mathrm{~mW} \cdot \mathrm{cm}^{-2}$, 且从 $50 \mathrm{~cm}$ 长截 下来的两段可以为LED灯供电(图15e)。

目前人们对于电缆构型的锌一空气电池探索 还相对较少, 而整体结构好、机械性能好的锌-空 气电池电化学性能又很差。开发出机械性能好、体 积小、电化学性能优异的锌-空气电池是未来的发 展趋势。运用各种原位生长技术如: 电沉积、气相 催化沉积、静电纺丝等技术, 可将催化剂与载体良 好的结合起来。为更好的提高水凝胶与电极之间 的附着性, 目前通常采用的解决方案是将水凝胶 电解质前驱体先涂抹在电极上, 随后凝固。同时将 空气阴极整合, 甚至是将空气阴极与电解质整合 到一起。而开发出新的在电极上进行原位生长电 解质的方法也亟待探索, 这也是能尽快将柔性锌空气电池运用到市场上的有效手段。

\section{8 总结}

综上所述, 近年来柔性锌-空气电池在实验室 的发展取得了重大进展。人们已经开发出了几种 不同结构的柔性锌-空气电池, 以提高不同变形下 的机械稳定性, 同时保持良好的电化学性能, 包括 高放电容量、高功率密度、高能量效率和长循环寿 命等。然而, 许多技术仍然存在着挑战(图16), 需 要在几个关键领域取得突破, 才能开发出电池性 能, 特别是耐久性显著提高的新一代柔性锌-空气 电池。

首先, 新型电池结构是满足电池机械灵活性 要求的关键。三明治式结构已经被广泛采用, 而值 得注意的设计是电缆式结构, 其具有极强的全方 位灵活性, 可以与纺织技术相结合, 促进可穿戴电 子设备的应用。但同时应注意的是, 在柔性电池的 实际使用中, 除了周围的气体环境外, 人肢体动作

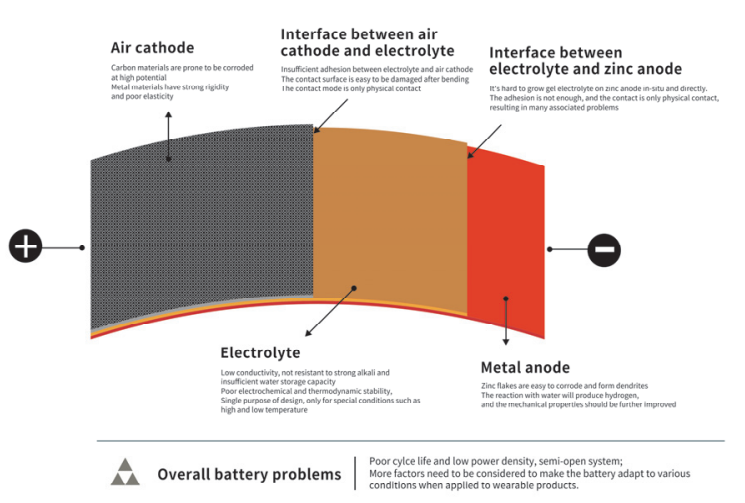

图 16 锌-空气电池存在的问题

Fig. 16 The challenges in zinc-air batteries. 
或器械拉伸带来的力学上的影响也应被考虑进 去, 将电化学与力学性能相结合, 才能制备出更可 靠的柔性储能设备。

其次, 目前的柔性锌-空气电池, 虽然功率较 高, 但循环性能较差, 循环性能好的功率密度又较 低, 做出使用时间长久且高效的锌-空气电池仍是 一个挑战。人们在描述各电池的机械性能与对应 的电池性能各有不同, 这就导致了并不能很好的 对目前电池存在的问题进行系统化的研究。因此, 在评估电池或电池组件的柔韧性时, 应采用更全 面的机械测试, 包括弯曲和/或扭向不同角度, 以 及测试长弯曲/拉伸循环下的稳定性。重要的是建 立一个标准的基准程序和参数, 以便将该技术与 其他储能系统进行公平的比较。同时, 需要更系统 的评估, 包括机械柔性和电化学性能。

进一步说, 柔性锌-空气电池包含三个部分: 凝胶电解质、柔性金属阳极以及柔性空气阴极。对 于凝胶电解质而言, 最基本的是要有较高的离子 电导率完成传导。其次, 凝胶电解质也要具备良好 的机械性能。在满足上述条件的同时要考虑电解 质与金属电极之间界面的问题，良好的接触可以 尽量减少枝晶的产生, 而电解质与空气阴极的界 面问题则更为复杂, 界面之间的接触不能紧密使 得 $\mathrm{OH}^{-}$离子的传导并不流畅。当然不仅仅包含这些 问题, 凝胶电解质需要良好的保水能力、高低温区 间工作的能力等各种因素也要考虑进去。

对于金属阳极来说, 除了要应对传统的锌枝 晶问题、碳酸盐沉积问题外, 同时也要考虑在形变 过程中遇到的各种问题, 例如弯折、扭曲、拉伸后 金属电极的功能遭到了破坏。对金属电极进行修 饰使其具备良好的机械性能, 同时其抗疲劳恢复 能力也是一个重要的指标。

最后对于空气阴极, 将催化剂与基底或集流 体整合到一起, 不仅能起到结构优化的作用甚至 起到了协同作用。整合后的空气阴极机械性能也 将大大提高, 避免在重复使用、拉伸、弯折的过程 中, 任意一方的脱落。在此基础上设计出催化性能 更好的催化剂则是一个次要目标。

总而言之, 尽管柔性锌-空气电池在柔性储能 系统中具有广阔的应用前景, 但其研究与开发仍 处于起步阶段, 还存在着许多技术障碍。其中, 最 关键的是缺乏对电池在机械外力作用下的耦合传 输和电化学过程的基本认识。它通常涉及一系列 沿表面、跨界面和通过复杂电极中的一些相的基 本电荷和传质过程, 这导致在确定整个过程步骤 方面存在困难。有必要在进行机械性能测试的同
时, 运用原位表征的技术观察微观结构的变化, 这 对于实现高性能柔性电池更好的材料和新型电池 配置的合理设计至关重要。柔性锌-空气电池未来 的发展也将有益于超级电容器或其他金属-空气 电池的学习与借鉴。人们的目光也要将其他柔性 储能器件所具备的优势尝试运用于柔性锌-空气 电池当中, 使其得到长足的发展。

\section{References}

(1) Guo, Y.; Li, H.; Zhai, T. Adv. Mater. 2017, 29, 1700007. doi: 10.1002/adma.201700007

(2) Ye, L.; Hong, Y.; Liao, M.; Wang, B.; Wei, D.; Peng, H.; Ye, L.; Hong, Y.; Liao, M.; Wang, B.; et al. Energy Storage Mater. 2020, 28 , 364. doi: 10.1016/j.ensm.2020.03.015

(3) Peng, X.; Wei, L.; Liu, Y.; Cen, T.; Ye, Z.; Zhu, Z.; Ni, Z.; Yuan, D. Energy Fuels 2020, 34, 8931. doi: 10.1021/acs.energyfuels.0c01167

(4) Lin, X.; Kang, Q.; Zhang, Z.; Liu, R.; Li, Y.; Huang, Z.; Feng, X.; Ma, Y.; Huang, W. J. Mater. Chem. A 2017, 5, 3638. doi: 10.1039/c6ta09806a

(5) Qin, R.; Wang, P.; Lin, C.; Cao, F.; Zhang, J.; Chen, L.; Mu, S. Acta Phys. -Chim. Sin. 2021, 37, 2009099. [秦睿, 王鹏彦, 林灿, 曹菲, 张金咏, 陈磊, 木士春. 物理化学学报, 2021, 37, 2009099.] doi: 10.3866/PKU.WHXB202009099

(6) Yang, B.; Yuan, W. ACS Appl. Mater. Interfaces 2019, 11, 16765. doi: 10.1021/acsami.9b01989

(7) Xu, Y.; Zhang, Y.; Guo, Z.; Ren, J.; Wang, Y.; Peng, H. Angew. Chem. Int. Ed. 2015, 54, 15390. doi: 10.1002/anie. 201508848

(8) Zhang, K.; Simic, R.; Yan, W.; Spencer, N. D. ACS Appl. Mater. Interfaces 2019, 11, 25427. doi: 10.1021/acsami.9b07387

(9) Karami, P.; Wyss, C. S.; Khoushabi, A.; Schmocker, A.; Broome, M.; Moser, C.; Bourban, P. E.; Pioletti, D. P. ACS Appl. Mater. Interfaces 2018, 10, 38692. doi: 10.1021/acsami.8b10735

(10) Gong, J. P.; Katsuyama, Y.; Kurokawa, T.; Osada, Y. Adv. Mater. 2003, 15, 1155. doi: 10.1002/adma.200304907

(11) Fotouhi, G.; Ogier, C.; Kim, J.-H.; Kim, S.; Cao, G.; Shen, A. Q.; Kramlich, J.; Chung, J.-H. J. Micromec. Microen. 2016, 26, 8. doi: 10.1088/0960-1317/26/5/055011

(12) Fan, X.; Liu, J.; Song, Z.; Han, X.; Deng, Y.; Zhong, C.; Hu, W. Nano Energy 2019, 56, 454. doi: 10.1016/j.nanoen.2018.11.057

(13) Zhao, S.; Xia, D.; Li, M.; Cheng, D.; Wang, K.; Meng, Y. S.; Chen, Z.; Bae, J. ACS Appl. Mater. Interfaces 2021, 13, 12033. doi: 10.1021/acsami.1c00012

(14) Li, M.; Liu, B.; Fan, X.; Liu, X.; Liu, J.; Ding, J.; Han, X.; Deng, Y.; Hu, W.; Zhong, C. ACS Appl. Mater. Interfaces 2019, 11, 28909. doi: 10.1021/acsami.9b09086

(15) Chen, R.; Xu, X.; Peng, S.; Chen, J.; Yu, D.; Xiao, C.; Li, Y.; Chen, 
Y.; Hu, X.; Liu, M.; et al. ACS Sustain. Chem. Eng. 2020, 8, 11501. doi: 10.1021/acssuschemeng.0c01111

(16) Ma, L.; Chen, S.; Wang, D.; Yang, Q.; Mo, F.; Liang, G.; Li, N.; Zhang, H.; Zapien, J. A.; Zhi, C. Adv. Energy Mater. 2019, 9, 46. doi: 10.1002/aenm.201803046

(17) Sun, N.; Lu, F.; Yu, Y.; Su, L.; Gao, X.; Zheng, L. ACS Appl. Mater. Interfaces 2020, 12, 11778. doi: 10.1021/acsami.0c00325

(18) Jiang, G.; Goledzinowski, M.; Comeau, F. J. E.; Zarrin, H.; Lui, G.; Lenos, J.; Veileux, A.; Liu, G.; Zhang, J.; Hemmati, S.; et al. Adv. Funct. Mater. 2016, 26, 1729.

doi: $10.1002 / \mathrm{adfm} .201504604$

(19) Gao, W.; Wu, G.; Janicke, M. T.; Cullen, D. A.; Mukundan, R.; Baldwin, J. K.; Brosha, E. L.; Galande, C.; Ajayan, P. M.; More, K. L.; et al. Angew. Chem. Int. Ed. 2014, 53, 3588. doi: 10.1002/anie. 201310908

(20) Zhang, J.; Fu, J.; Song, X.; Jiang, G.; Zarrin, H.; Xu, P.; Li, K.; Yu, A.; Chen, Z. Adv. Energy Mater. 2016, 6, 476. doi: 10.1002/aenm.20160047620.

(21) Song, Z.; Ding, J.; Liu, B.; Liu, X.; Han, X.; Deng, Y.; Hu, W.; Zhong, C. Adv. Mater. 2020, 32, e1908127. doi: 10.1002/adma.201908127

(22) Haynes, W. M. CRC Handbook of Chemistry and Physics, 97th ed.; CRC Press: Boca Raton, FL, USA, 2017; pp. 5-17.

(23) Fu, J.; Cano, Z. P.; Park, M. G.; Yu, A.; Fowler, M.; Chen, Z. Adv. Mater. 2017, 29, 1604685. doi: 10.1002/adma.201604685

(24) Shin, H.-J.; Kwak, W.-J.; Aurbach, D.; Sun, Y.-K. Adv. Funct. Mater. 2017, 27, 1605500. doi: 10.1002/adfm.201605500

(25) Banik, S. J.; Akolkar, R. J. Electrochem. Soc. 2013, 160, D519. doi: $10.1149 / 2.040311 \mathrm{jes}$

(26) Khezri, R.; Hosseini, S.; Lahiri, A.; Motlagh, S. R.; Nguyen, M. T.; Yonezawa, T.; Kheawhom, S. Int. J. Mol. Sci. 2020, 21,7303. doi: $10.3390 /$ ijms 21197303

(27) Yang, X.; Wu, X.; Guo, Z.; Li, Q.; Wang, H.; Ke, C.; Zeng, W.; Qiu, X.; He, Y.; Liang, X.; et al. RSC Adv. 2020, 10, 33327. doi: $10.1039 / \mathrm{d} 0 \mathrm{ra} 04827 \mathrm{e}$
(28) Pan, Z.; Yang, J.; Zang, W.; Kou, Z.; Wang, C.; Ding, X.; Guan, C.; Xiong, T.; Chen, H.; Zhang, Q.; et al. Energy Storage Mater. 2019, 23, 375. doi: 10.1016/j.ensm.2019.04.036

(29) Qu, S.; Liu, B.; Wu, J.; Zhao, Z.; Liu, J.; Ding, J.; Han, X.; Deng, Y.; Zhong, C.; Hu, W. ACS Appl. Mater. Interfaces 2020, 12, 54833. doi: 10.1021/acsami.0c17479

(30) Wang, Z.; Meng, X.; Wu, Z.; Mitra, S. J. Energy Chem. 2017, 26, 129. doi: 10.1016/j.jechem.2016.08.007

(31) Fu, J.; Lee, D. U.; Hassan, F. M.; Yang, L.; Bai, Z.; Park, M. G.; Chen, Z. Adv. Mater. 2015, 27, 5617. doi: 10.1002/adma.201502853

(32) Kangasniemi, K. H.; Condit, D. A.; Jarvi, T. D. J. Electrochem. Soc. 2004, 151, E125. doi: 10.1149/1.1649756

(33) Wang, X.; Li, W.; Chen, Z.; Waje, M.; Yan, Y. J. Power Sources. 2006, 158, 154. doi: 10.1016/j.jpowsour.2005.09.039

(34) Xu, N.; Wilson, J. A.; Wang, Y.-D.; Su, T.; Wei, Y.; Qiao, J.; Zhou, X.-D.; Zhang, Y.; Sun, S. Appl. Catal. B. 2020, 272, 118953. doi: 10.1016/j.apcatb.2020.118953

(35) Wu, M.; Zhang, G.; Chen, N.; Chen, W.; Qiao, J.; Sun, S. Energy Storage Mater. 2020, 24, 272. doi: 10.1016/j.ensm.2019.08.009

(36) Yang, X.; Wu, X.; Guo, Z.; Li, Q.; Wang, H.; Ke, C.; Zeng, W.; Qiu, X.; He, Y.; Liang, X.; et al. RSC Adv. 2020, 10, 33327. doi: $10.1039 / \mathrm{d} 0 \mathrm{ra} 04827 \mathrm{e}$

(37) Tan, Y.; Zhang, Z.; Lei, Z.; Wu, W.; Zhu, W.; Cheng, N.; Mu, S. J. Power Sources 2020, 473, 228570. doi: 10.1016/j.jpowsour.2020.228570

(38) Lei, Z.; Tan, Y.; Zhang, Z.; Wu, W.; Cheng, N.; Chen, R.; Mu, S.; Sun, X. Nano Res. 2020, 14, 868. doi: 10.1007/s12274-020-3127-8

(39) Zhang, W.; Li, Z.; Chen, J.; Wang, X.; Li, X.; Yang, K.; Li, L. Nanotechnology 2020, 31, 185703. doi: 10.1088/1361-6528/ab6cd9

(40) Park, J.; Park, M.; Nam, G.; Lee, J. S.; Cho, J. Adv. Mater. 2015, 27 , 1396. doi: 10.1002/adma.201404639

(41) Li, S.; Yang, X.; Yang, S.; Gao, Q.; Zhang, S.; Yu, X.; Fang, Y.; Yang, S.; Cai, X. J. Mater. Chem. A 2020, 8, 5601. doi: 10.1039/d0ta00888e

(42) Zeng, S.; Tong, X.; Zhou, S.; Lv, B.; Qiao, J.; Song, Y.; Chen, M.; Di, J.; Li, Q. Small 2018, 14, e1803409. doi: 10.1002/smll.20180340 